\title{
British Society of Gastroenterology guidelines on the management of irritable bowel syndrome
}

\author{
Dipesh H Vasant (1) , 1,2 Peter A Paine, ${ }^{2,3}$ Christopher J Black (1) , ${ }^{4,5}$ \\ Lesley A Houghton 지, ${ }^{5,6}$ Hazel A Everitt, ${ }^{7}$ Maura Corsetti, ${ }^{8}$ Anurag Agrawal, ${ }_{1}^{9}$ \\ Imran Aziz (10 , ${ }^{10,11}$ Adam D Farmer, ${ }^{12,13}$ Maria P Eugenicos, ${ }^{14}$ Rona Moss-Morris, ${ }^{15}$ \\ Yan Yiannakou, ${ }^{16}$ Alexander C Ford (i) ${ }^{4,5}$
}

- Additional supplemental material is published online only. To view, please visit the journal online (http://dx.doi.org/ 10.1136/gutjnl-2021-324598)

For numbered affiliations see end of article.

\section{Correspondence to} Professor Alexander C Ford, Leeds Gastroenterology Institute, St James's University Hospital, Leeds, UK alexf12399@yahoo.com

DHV and PAP are joint first authors.

Received 6 March 2021 Revised 30 March 2021 Accepted 6 April 2021 Published Online First 26 April 2021

\section{Check for updates}

(c) Author(s) (or their employer(s)) 2021. No commercial re-use. See rights and permissions. Published by BMJ.

To cite: Vasant $\mathrm{DH}$, Paine PA Black CJ, et al. Gut

2021:70:1214-1240.

\section{ABSTRACT}

Irritable bowel syndrome (IBS) remains one of the most common gastrointestinal disorders seen by clinicians in both primary and secondary care. Since publication of the last British Society of Gastroenterology (BSG) guideline in 2007, substantial advances have been made in understanding its complex pathophysiology, resulting in its re-classification as a disorder of gut-brain interaction, rather than a functional gastrointestinal disorder. Moreover, there has been a considerable amount of new evidence published concerning the diagnosis, investigation and management of IBS. The primary aim of this guideline, commissioned by the BSG, is to review and summarise the current evidence to inform and guide clinical practice, by providing a practical framework for evidence-based management of patients. One of the strengths of this guideline is that the recommendations for treatment are based on evidence derived from a comprehensive search of the medical literature, which was used to inform an update of a series of trial-based and network meta-analyses assessing the efficacy of dietary, pharmacological and psychological therapies in treating IBS. Specific recommendations have been made according to the Grading of Recommendations Assessment, Development and Evaluation system, summarising both the strength of the recommendations and the overall quality of evidence. Finally, this guideline identifies novel treatments that are in development, as well as highlighting areas of unmet need for future research.

\section{EXECUTIVE SUMMARY OF RECOMMENDATIONS Doctor-patient communication}

- Establishing an effective doctor-patient relationship and a shared understanding is key to the management of IBS. Such a relationship can lead to improved quality of life and symptoms, reduce healthcare visits and enhance adherence to treatment (recommendation: strong, quality of evidence: low).

- Patients with IBS would like increased empathy, support and information from clinicians about the nature of the condition, diagnosis and symptom management options (recommendation: strong, quality of evidence: low).

\section{Diagnosis, investigation and education}

- The National Institute for Health and Care Excellence guideline definition of IBS (abdominal pain or discomfort, in association with altered bowel habit, for at least 6 months, in the absence of alarm symptoms or signs) is more pragmatic and may be more applicable to patients with IBS in primary care than diagnostic criteria derived from patients in secondary care, such as the Rome IV criteria (recommendation: weak, quality of evidence: low).

- All patients presenting with symptoms of IBS for the first time in primary care should have a full blood count, $\mathrm{C}$ reactive protein or erythrocyte sedimentation rate, coeliac serology and, in patients $<45$ years of age with diarrhoea, a faecal calprotectin to exclude inflammatory bowel disease. Local and national guidelines for colorectal and ovarian cancer screening should be followed, where indicated (recommendation: strong, quality of evidence: moderate).

- Clinicians should make a positive diagnosis of IBS based on symptoms, in the absence of alarm symptoms or signs, and abnormalities on simple blood and stool tests (recommendation: strong, quality of evidence: moderate).

- Referral to gastroenterology in secondary care is warranted where there is diagnostic doubt, in patients with symptoms that are severe, or refractory to first-line treatments, or where the individual patient requests a specialist opinion (recommendation: weak, quality of evidence: low).

- There is no role for colonoscopy in IBS, other than in those with alarm symptoms or signs, or those with symptoms suggestive of IBS with diarrhoea who have atypical features and/or relevant risk factors that increase the likelihood of them having microscopic colitis (female sex, age $\geq 50$ years, coexistent autoimmune disease, nocturnal or severe, watery, diarrhoea, duration of diarrhoea $<12$ months, weight loss or use of potential precipitating drugs including non-steroidal anti-inflammatory drugs, proton pump inhibitors, etc) (recommendation: strong, quality of evidence: moderate).

- In those with symptoms suggestive of IBS with diarrhoea, but with atypical features such as nocturnal diarrhoea, or a prior cholecystectomy, 23-seleno-25-homotaurocholic acid scanning or serum $7 \alpha$-hydroxy-4-cholesten3 -one should be considered to exclude bile acid 
diarrhoea (recommendation: strong, quality of evidence: low).

- In patients with IBS and coexisting symptoms suggestive of a defaecatory disorder or faecal incontinence, anorectal physiology tests can be considered, where available, to select those who might benefit from biofeedback (recommendation: weak, quality of evidence: low).

- There is no role for testing for exocrine pancreatic insufficiency, or for hydrogen breath testing to rule out small intestinal bacterial overgrowth or carbohydrate intolerance, in patients with typical IBS symptoms (recommendation: strong, quality of evidence: weak).

- The diagnosis of IBS, its underlying pathophysiology and the natural history of the condition, including common symptom triggers, should be explained to the patient. This should introduce the concept of IBS as a disorder of gutbrain interaction, together with a simple account of the gutbrain axis and how this is impacted by diet, stress, cognitive, behavioural and emotional responses to symptoms, and postinfective changes (recommendation: strong, quality of evidence: weak).

\section{First-line treatments}

- All patients with IBS should be advised to take regular exercise (recommendation: strong, quality of evidence: weak).

- First-line dietary advice should be offered to all patients with IBS (recommendation: strong, quality of evidence: weak).

- Food elimination diets based on IgG antibodies are not recommended in patients with IBS (recommendation: strong, quality of evidence: moderate).

- Soluble fibre, such as ispaghula, is an effective treatment for global symptoms and abdominal pain in IBS, but insoluble fibre (eg, wheat bran) should be avoided as it may exacerbate symptoms. Soluble fibre should be commenced at a low dose (3-4 g/day) and built up gradually to avoid bloating (recommendation: strong; quality of evidence: moderate).

- A diet low in fermentable oligosaccharides, disaccharides and monosaccharides and polyols, as a second-line dietary therapy, is an effective treatment for global symptoms and abdominal pain in IBS, but its implementation should be supervised by a trained dietitian and fermentable oligosaccharides, disaccharides and monosaccharides and polyols should be reintroduced according to tolerance (recommendation: weak, quality of evidence very low).

- A gluten-free diet is not recommended in IBS (recommendation: weak, quality of evidence very low).

- Probiotics, as a group, may be an effective treatment for global symptoms and abdominal pain in IBS, but it is not possible to recommend a specific species or strain. It is reasonable to advise patients wishing to try probiotics to take them for up to 12 weeks, and to discontinue them if there is no improvement in symptoms (recommendation: weak, quality of evidence: very low).

- Loperamide may be an effective treatment for diarrhoea in IBS. However, abdominal pain, bloating, nausea and constipation are common, and may limit tolerability. Titrating the dose carefully may avoid this (recommendation: strong; quality of evidence: very low).

- Certain antispasmodics may be an effective treatment for global symptoms and abdominal pain in IBS. Dry mouth, visual disturbance and dizziness are common side effects (recommendation: weak, quality of evidence: very low).
- Peppermint oil may be an effective treatment for global symptoms and abdominal pain in IBS. Gastro-oesophageal reflux is a common side effect (recommendation: weak, quality of evidence: very low).

- Polyethylene glycol may be an effective treatment for constipation in IBS. Abdominal pain is a common side effect (recommendation: weak; quality of evidence: very low).

\section{Second-line treatments}

- Tricyclic antidepressants used as gut-brain neuromodulators are an effective second-line drug for global symptoms and abdominal pain in IBS. They can be initiated in primary or secondary care, but careful explanation as to the rationale for their use is required, and patients should be counselled about their side-effect profile. They should be commenced at a low dose (eg, $10 \mathrm{mg}$ amitriptyline once a day) and titrated slowly to a maximum of 30-50 mg once a day (recommendation: strong, quality of evidence: moderate).

- Selective serotonin reuptake inhibitors used as gut-brain neuromodulators may be an effective second-line drug for global symptoms in IBS. As with tricyclic antidepressants, they can be initiated in primary or secondary care, but careful explanation as to the rationale for their use is required, and patients should be counselled about their side-effect profile (recommendation: weak, quality of evidence: low).

- Eluxadoline, a mixed opioid receptor drug, is an efficacious second-line drug for IBS with diarrhoea in secondary care. It is contraindicated in patients with prior sphincter of Oddi problems or cholecystectomy, alcohol dependence, pancreatitis or severe liver impairment, and lack of availability may limit its use (recommendation: weak, quality of evidence: moderate).

- 5-Hydroxytryptamine 3 receptor antagonists are efficacious second-line drugs for IBS with diarrhoea in secondary care. Alosetron and ramosetron are unavailable in many countries; ondansetron titrated from a dose of $4 \mathrm{mg}$ once a day to a maximum of $8 \mathrm{mg}$ three times a day is a reasonable alternative. Constipation is the most common side effect. This drug class is likely the most efficacious for IBS with diarrhoea (recommendation: weak, quality of evidence: moderate to high).

- The non-absorbable antibiotic rifaximin is an efficacious second-line drug for IBS with diarrhoea in secondary care, although its effect on abdominal pain is limited. The drug is licensed for IBS with diarrhoea in the USA but is not available for this indication in many countries (recommendation: weak, quality of evidence: moderate).

- Linaclotide, a guanylate cyclase-C agonist, is an efficacious second-line drug for IBS with constipation in secondary care. It is likely to be the most efficacious secretagogue available for IBS with constipation, although diarrhoea is a common side effect (recommendation: strong, quality of evidence: high).

- Lubiprostone, a chloride channel activator, is an efficacious second-line drug for IBS with constipation in secondary care. This secretagogue is less likely to cause diarrhoea than others. However, patients should be warned that nausea is a frequent side effect (recommendation: strong, quality of evidence: moderate).

- Plecanatide, another guanylate cyclase-C agonist, is an efficacious second-line drug for IBS with constipation in secondary care. Diarrhoea is a common side effect and is no less likely than with linaclotide or tenapanor. Although 
the drug is licensed for IBS with constipation in the USA, it is not yet available for this indication in many countries (recommendation: strong, quality of evidence: high).

- Tenapanor, a sodium-hydrogen exchange inhibitor, is an efficacious second-line drug for IBS with constipation in secondary care. Again, diarrhoea is a frequent side effect. Although the drug is licensed for IBS with constipation in the USA, it is not yet available for this indication in many countries (recommendation: strong, quality of evidence: high).

- Tegaserod, a 5-Hydroxytryptamine 4 receptor agonist, is an efficacious second-line drug for IBS with constipation in secondary care but is unavailable outside the USA. Diarrhoea is a common side effect (recommendation: strong, quality of evidence: moderate).

\section{Psychological therapies}

- IBS-specific cognitive behavioural therapy may be an efficacious treatment for global symptoms in IBS (recommendation: strong, quality of evidence: low).

- Gut-directed hypnotherapy may be an efficacious treatment for global symptoms in IBS (recommendation: strong, quality of evidence: low).

- Psychological therapies should be considered when symptoms have not improved after 12 months of drug treatment. Referral can be made at an earlier stage, if accessible locally, and based on patient preference (recommendation: strong, quality of evidence: low).

\section{Management of severe or refractory IBS}

- Severe or refractory IBS symptoms should prompt a review of the diagnosis, with consideration of further targeted investigation (recommendation: weak, evidence: very low).

- Severe or refractory IBS should be managed with an integrated multi-disciplinary approach (recommendation: weak, evidence: very low).

- Iatrogenic harms due to opioid prescribing, unnecessary surgery and unproven unregulated diagnostic or therapeutic approaches incentivised by financial or reputational gain should be avoided (recommendation: strong, evidence: very low).

- Use of combination gut-brain neuromodulators, termed augmentation, may be considered for more severe symptoms, with vigilance for risks of serotonin syndrome (recommendation: weak, evidence: very low).

\section{Research}

- Successful completion of large clinical trials will require pragmatic inclusion criteria, minimisation of the participant trial burden and effective recruitment strategies that reach into community settings. Virtual (remote access) trial approaches will reduce geographical exclusion.

- A priority-setting partnership would best discern valuable research questions.

- Some future research themes include, but are not limited to: - Characterisation of the illness to understand predictors (clinical, genetic, psychological and biological) of outcome and treatment response, determinants of refractory illness and burden of illness (particularly with respect to workplace productivity) by conducting large-scale epidemiological studies with extended observation.

- Trials of novel treatments, including pharmacological, dietary and behavioural therapies, device-based treatments and faecal microbiota transplantation. There is also a need for development of visceral analgesics. Consideration should be given to stratifying randomised controlled trials by IBS severity and subtype, burden of extraintestinal symptoms and psychological comorbidity.

- A better understanding of treatment combinations to uncover augmentation effects between therapies, and to assess the value of multidisciplinary approaches.

- Modulation of pain and psychological responses using pharmacological (eg, serotonin norepinephrine reuptake inhibitors) or behavioural approaches (eg, cognitive behavioural therapy used earlier in the disease course or via digital provision), and comparison of cognitive behavioural therapy with gut-directed hypnotherapy.

- Med-tech approaches (web-based, apps and devices) to behavioural modification.

\section{Patient summary}

These guidelines have been produced on behalf of the British Society of Gastroenterology (BSG) by a team of specialists, and with input from patients with irritable bowel syndrome (IBS). The guidelines are aimed at healthcare professionals who look after patients with IBS.

IBS is a common condition, which is caused by problems arising between the gut and the brain. It consists of symptoms like abdominal pain linked to changes in bowel frequency or appearance of stools, and often bloating. Problems in IBS have been found in the nervous system supplying the gut, often making it more sensitive. Psychological factors (including stress), certain foods and the micro-organisms (bugs) living in the gut can all play a role in triggering symptoms. Occasionally, it can start after a gut infection, or antibiotic use, but more often there is no clear origin.

Some patients with IBS learn to manage their symptoms themselves, by changing their lifestyle or diet, or managing stress differently. Others, however, will consult their general practitioner who can usually make the diagnosis based on the typical symptoms. General practitioners will carry out some blood tests, including one to rule out coeliac disease (an immune reaction to gluten) and, if diarrhoea is present, a stool test to rule out inflammation. If there are concerning symptoms, including bleeding from the back passage, substantial weight loss or anaemia, a strong family history of cancer, or the patient is older, then the general practitioner will refer to a hospital specialist for further tests. The specialist may request a camera test of the large bowel, known as a colonoscopy, or do extra tests to look for other causes of diarrhoea or constipation, especially if the patient's symptoms are less typical of IBS.

Regular exercise, making some simple dietary changes, and adopting healthy eating patterns will help many patients. Some patients find reducing dietary fibre improves symptoms, while others may find that a soluble fibre supplement helps. Referral to a dietitian can be helpful if these first-line approaches to diet do not help. Taking supplements of probiotics (often referred to as 'friendly bacteria') may also help, but these can be expensive.

Some patients may require different medications, depending on their main symptom. Some of these can be obtained over the counter, but others need to be prescribed by a doctor. This guideline has reviewed the evidence for which medications work, and the possible harms they may cause. We have only recommended medications with good evidence that they are effective and have recommended against tests or treatments where the evidence is that they do not help, are harmful or where there is not enough 
evidence. Some medications have most of their effect on the gut itself, others work both at the level of the gut and the brain (called 'neuromodulators' as they help to reduce nerve sensitivity). Some drugs that have good evidence are unfortunately not available, or are too expensive, in some countries.

There is good evidence that psychological treatments directed against IBS symptoms, especially cognitive behavioural therapy (CBT), and hypnotherapy, are helpful for many patients' symptoms, but unfortunately these are not always readily accessible. Work is being done to improve access to these.

Very severe symptoms that do not respond to some of the above treatments are rare. However, patients whose symptoms do not improve may be left feeling desperate, and therefore vulnerable to approaches which are not proven, expensive or high risk. It is recommended that patients in this position are supported by a multidisciplinary specialist team to help reduce harms, such as unwarranted tests or operations, or harmful drugs.

Although much progress has been made in understanding and treating IBS, there are still many things we do not know about the condition, and there are lots of active areas for research, and therapies that need to be explored. We hope this guideline will also help to highlight and prioritise these areas.

\section{INTRODUCTION \\ Aims}

Since the last BSG guideline on IBS was published in $2007,{ }^{1}$ there has been a considerable amount of new evidence pertaining to the pathophysiology, diagnosis, investigation and management of the condition. Furthermore, the gold standard symptombased diagnostic criteria for IBS, the Rome criteria, are now in their fourth iteration. ${ }^{2}$ The primary aim of this guideline, commissioned by the BSG, is to update the 2007 guideline, considering all these developments, but with a particular focus on treatment of the condition. The overarching intention is to provide a guideline that is practical to use and an authoritative framework for current, state-of-the art, evidence-based clinical practice.

\section{Methodology}

In line with the Appraisal of Guidelines for REsearch \& Evaluation guideline development protocol, ${ }^{3}$ a diverse multidisciplinary working group of clinicians and academics was convened from across the interface of primary, secondary and tertiary care, as well as psychology and dietetics. To ensure a patient-centred approach at the outset, the proposal was reviewed by the IBS network, Guts UK, and by four patients with IBS who were invited to join the working group.

Each section lead performed a comprehensive literature search, except for the section dealing with treatment, which was informed by a systematic review of the literature, the methodology for which is reported within that section. Eligible studies were graded according to the Oxford Centre for Evidence Based Medicine. ${ }^{4}$ The Grading of Recommendations Assessment, Development and Evaluation system was used to evaluate the strength of the recommendations and the overall quality of evidence. ${ }^{5}$ Thereafter, all members of the working group reviewed and approved the entire guideline.

\section{Conflicts of interest}

All members of the working group were asked to complete conflicts of interest declarations. These are available as a supplementary online table.

\section{Scheduled review}

We would suggest these guidelines are reviewed and updated every 4 years.

\section{CLASSIFICATION AND DIAGNOSTIC CRITERIA}

In the absence of any biomarker being available for IBS, the condition is diagnosed using a positive approach, based on the clinical history. Symptom-based diagnostic criteria have been developed to facilitate this, according to a specific pattern of gastrointestinal symptoms reported by the patient, with recourse to limited investigations. These criteria were developed by the Rome Foundation, and the most recent iteration, Rome IV, were published in 2016 (table 1). ${ }^{2}$ These define IBS as the presence of abdominal pain, related to defaecation, associated with a change in stool frequency and/or stool form. Patients are subgrouped according to their predominant stool pattern into IBS with diarrhoea (IBS-D), IBS with constipation (IBS-C), IBS with mixed bowel habits (IBS-M) or IBS unclassified (IBS-U), to direct therapy. The presence of abdominal pain at the required frequency distinguishes IBS from the other functional bowel disorders, which consist of functional constipation, functional diarrhoea and functional abdominal bloating or distension. ${ }^{2}$ However, there is some degree of overlap and fluctuation between IBS and these other disorders. ${ }^{67}$ The Rome IV process also redefined IBS as a disorder of gut-brain interaction, in recognition of the complex interplay of biological, psychological, and social factors underpinning the condition.

The Rome IV criteria made some important changes, ${ }^{2}$ compared with their predecessor, Rome III. ${ }^{8}$ First, abdominal 'discomfort' was removed from the definition, as this was felt to be a vague term that was not understandable in some languages. ${ }^{9}$ Second, the minimum required frequency of abdominal pain was increased from at least 3 days per month, to at least 1 day per week. This change reflected the findings of a normative survey showing that adopting a higher threshold for the frequency of abdominal pain required to meet criteria for IBS would lead to fewer healthy people in the general population being misclassified as having IBS, ${ }^{9}$ and therefore risk being 'medicalised', and having to take drugs for the condition. This feature makes the Rome IV criteria potentially more specific than Rome III. Third, it was no longer necessary for abdominal pain to be relieved by defaecation. Instead, it should be 'related to defaecation', acknowledging that some patients with IBS report that their pain worsens following a bowel movement. ${ }^{2}$

This more restrictive nature of the Rome IV criteria calls into question whether they should be used to diagnose IBS in clinical practice, and a more pragmatic definition of the symptoms that constitute IBS may be preferred. The National Institute for Health and Care Excellence (NICE) guideline for the management of IBS in primary care recommends a broader, more pragmatic, definition of IBS, ${ }^{10}$ focusing on abdominal pain or discomfort associated with altered stool frequency or stool form for at least 6 months, in the absence of alarm symptoms or signs, and acknowledging that coexistent bloating, lethargy, nausea, backache or bladder symptoms are common.

\section{EPIDEMIOLOGY}

Symptoms compatible with IBS are extremely common in the general population at any particular point in time, ${ }^{11}$ and experienced on a continuum, from what may be fleeting and part of normal health, to a disease process, requiring medical input. In the latter instance, IBS is chronic, with fluctuating symptoms, in the majority of patients. ${ }^{12}$ However, few epidemiological studies 
have conducted follow-up beyond 12 months, and most drug trials take place over 12 weeks, meaning that factors predicting continuation or resolution of symptoms is unclear. The condition impacts on social functioning, quality of life ${ }^{13}$ and ability to work, with one-in-four patients reporting sickness-related absences from work, and up to $80 \%$ presenteeism in the workplace. ${ }^{14}$ The annual direct and indirect costs related to IBS are estimated to be up to €8 billion in Europe, ${ }^{15} ¥ 123$ billion in China $^{16}$ and in excess of US $\$ 10$ billion in the USA. ${ }^{17}$

Prior to the publication of the Rome IV criteria in 2016, 2 systematic reviews and meta-analyses quantified the global prevalence of IBS. The first of these, published in 2012, included data from 260960 individuals across 81 different countries and calculated a pooled global prevalence of IBS of $11 \%$, irrespective of definition used. ${ }^{11}$ Prevalence varied widely between countries, and according to the criteria used to define IBS, ranging from $1.1 \%$ in one Iranian study that used the Rome III criteria, to $45 \%$ in a study from Pakistan that used Rome II. The second systematic review and meta-analysis, from 2017 and conducted by the Rome Foundation, found similar variability in the prevalence of IBS, which ranged from 1.1\% in France and Iran, to $35.5 \%$ in Mexico, and with a pooled global prevalence of $8.8 \%{ }^{18}$ In both meta-analyses, heterogeneity between studies was substantial, presumably relating to differences in methodology, demographic characteristics of participants, cultural issues or a combination of these factors.

Due to the uncertainty surrounding pooled estimates of global prevalence, and the apparent variation in prevalence between countries in separate studies, a subsequent Rome Foundation global survey has quantified the prevalence of IBS, among 73 000 adults in 33 different countries simultaneously, using both the Rome III and IV criteria. ${ }^{19}$ The worldwide prevalence of IBS was $4.1 \%$ using the Rome IV criteria, compared with $10.1 \%$ with Rome III. An update of the systematic review and metaanalysis from 2012 incorporating the results of this global survey, ${ }^{20}$ demonstrated a pooled prevalence of IBS according to the Rome III criteria of $9.2 \%$, in 53 studies recruiting 400 000 participants from 38 countries, compared with $3.8 \%$ using Rome IV, based on findings from 6 studies including over 80000 individuals from 34 countries. With respect to sex, the prevalence of IBS was modestly, but significantly, higher in women than men in this meta-analysis (odds ratio (OR) 1.46; 95\% CI 1.33 to 1.59 ) based on 30 studies using the Rome III criteria. ${ }^{20}$ The Rome Foundation global survey also reported a substantially higher pooled prevalence of IBS among women, compared with men, using the Rome IV criteria (OR 1.8; 95\% CI 1.7 to 2.0). ${ }^{19}$ Prevalence of IBS decreased modestly with increasing age, irrespective of diagnostic criteria, in a prior meta-analysis, although this trend was not statistically significant. ${ }^{11}$ Similarly, in the Rome Foundation global survey, prevalence of both Rome III and Rome IV IBS decreased with age and was highest among adults aged $18-39$ years. ${ }^{19}$

The fall in prevalence in IBS that results from the changes made in moving from the Rome III to Rome IV criteria is noteworthy, reflecting the more restrictive nature of the latter. This has important clinical implications because, although as intended, the criteria are now more specific for diagnosing IBS, ${ }^{21}$ up to $50 \%$ of patients who believe they have IBS will no longer meet criteria for the condition. Instead, they will be diagnosed as having another functional bowel disorder, ${ }^{22}{ }^{23}$ such as functional diarrhoea, functional constipation or functional abdominal bloating or distension. Moreover, there may be an impact on treatment trials in IBS, and the interpretation of results, because patient populations recruited using the Rome IV criteria will 
differ from those recruited using Rome III, and may have more severe symptoms and higher degrees of psychological comorbidity. ${ }^{23}{ }^{24}$ Moving from Rome III to Rome IV IBS may therefore reduce the likelihood of novel pharmacological therapies demonstrating efficacy in future randomised controlled trials (RCTs), due to the spectrum of symptom severity, or may mean that trials need to be considerably larger, and therefore more expensive to conduct, to show a beneficial effect.

\section{PATHOPHYSIOLOGY}

The pathophysiology of IBS is complex and remains poorly understood. ${ }^{2}$ Genetics, and epigenetic changes, infection and early adverse life events may predispose an individual to developing IBS, ${ }^{25-28}$ and chronic stress, psychological symptoms, negative beliefs about symptoms and illness and maladaptive coping mechanisms can increase the frequency and severity of symptoms. ${ }^{29}{ }^{30}$ For some patients with IBS, psychological comorbidity or distress may be a consequence, rather than a cause, of the severity and frequency of symptoms experienced. ${ }^{31}$ IBS is a disorder of altered bidirectional communication between the gut and brain (via the gut-brain axis), and has a biopsychosocial aetiology. ${ }^{32}$ As a result, it has been re-termed a disorder of gut-brain interaction. ${ }^{32}$ An exhaustive discussion of the involved pathophysiological mechanisms is beyond the scope of this guideline, but the best accepted of these are summarised below.

\section{Central nervous system and autonomic nervous system modulation}

Symptoms are generated by interoceptive signals from the gut, and memories of such signals, and are modulated by emotional (anxiety, depression), cognitive (attention, beliefs and expectation) and motivational factors. ${ }^{33}$ Altered activation of brain regions responsible for cognitive processing and emotional and autonomic responses to visceral and somatic stimuli are seen in IBS, consistent with visceral hypersensitivity, hypervigilance and symptom-related anxiety. ${ }^{34}$ Some patients with IBS have compromised central inhibitory regulation of visceral and somatic stimuli. ${ }^{35}$ Modulating activity of the brain regions responsible for visceral pain using various therapies has been shown to improve IBS symptomatology. ${ }^{36-38}$

The autonomic nervous system mediates communication between the gut and brain. In IBS, a reduction in parasympathetic activity and an increase in sympathetic nervous system activity is frequently observed. ${ }^{39}$ Reduced vagal tone may be caused by stress, and impacts on gut motility and sensitivity, and peripheral inflammation and gut permeability. ${ }^{40}$ Conversely, the vagus nerve may sense the gut microenvironment indirectly and transfer this information to the brain.

\section{Altered visceral perception}

Between $20 \%$ and $60 \%$ of patients with IBS have enhanced visceral perception to various physiological stimuli (eg, mechanical or electrical). ${ }^{41}$ Hypersensitivity to mechanical distension of the gut is reported by more patients with IBS-D than IBS-C. However, studies do not distinguish between affective, cognitive and true peripheral, versus central, mechanisms of visceral hypersensitivity. A study conducted in separate patient cohorts from Sweden, Belgium and the USA reported that visceral sensitivity correlated positively with symptom severity, even after adjustment for the tendency to report symptoms or psychological comorbidity. ${ }^{42}$ Approximately $20 \%$ of patients with IBS are viscerally hyposensitive or insensitive to mechanical distension, more commonly those with IBS-C than IBS-D, with one study suggesting that the degree of insensitivity correlated with abdominal distension (ie, a true physical increase in abdominal girth). ${ }^{43}$

\section{Transit and motility}

Colonic transit is abnormal in only $10 \%-20 \%$ of patients with IBS-C and IBS-M, and $25 \%-45 \%$ of patients with IBSD. $^{44} 45$ Similar observations are seen for oro-caecal transit. ${ }^{46} 47$ However, patients with normal transit can still have abnormal fasting and postprandial motility. ${ }^{48}$ Patients with IBS-C display reduced motility, fewer high amplitude propagating contractions of the colon and delayed transit, whereas those with IBS-D have increased motility, more high amplitude propagating contractions and accelerated transit. ${ }^{1}$ Colonic transit time correlates inversely with stool consistency and, to a lesser extent, with stool frequency. ${ }^{45}$ However, symptoms of abdominal pain, bloating and flatulence correlate poorly, or not at all, with colonic transit, ${ }^{45}{ }^{46}$ whereas abdominal distension (the physical increase in abdominal girth, rather than the sensation of bloating) correlates with oro-caecal and colonic transit times, and inversely with stool consistency. ${ }^{46}$ High amplitude propagating contractions in patients with IBS-D are associated with abdominal pain. ${ }^{1}$ Changes in gastrointestinal motility may be influenced by alterations in serotonin (5-Hydroxytryptamine $(5-\mathrm{HT}))$ metabolism, ${ }^{49}$ with high levels reported in patients with IBS-D, and low levels in IBS-C. ${ }^{50}$

\section{Immune regulation, inflammation and epithelial permeability}

Low-grade mucosal inflammation may arise from a compromised epithelial barrier, dysbiosis or altered stress levels, and impaired epithelial barrier function from an aberrant stress and immune response, and/or dysbiosis. It can be linked to a previous episode of infectious gastroenteritis induced by bacteria, parasites or viruses, referred to as postinfection IBS (PI-IBS). ${ }^{51}$ Increased numbers of mast cells, particularly in the descending colon and rectosigmoid region, ${ }^{5253}$ and in the small intestine, ${ }^{54}$ is the most consistent histological finding in IBS. Mast cell hyperplasia is more common in IBS-D and PI-IBS. ${ }^{52} 55$ The severity and frequency of abdominal pain correlates with the presence of activated mast cells in close proximity to nerve endings in the gut mucosa in some studies, ${ }^{56}$ but in others mast cell proliferation associates with a reduction, ${ }^{57}$ or no change, ${ }^{58}$ in visceral sensitivity. Interleukin-10 mRNA expression and protein levels are consistently reduced in the mucosa and/or peripheral circulation, in patients with IBS-D and PI-IBS, and associate with comorbid anxiety or depression. ${ }^{59}$ There is lack of consensus on whether numbers of $\mathrm{T}$ cells and levels of cytokines in the peripheral circulation of patients with IBS are abnormal. ${ }^{5253}$

Increased permeability in patients with PI-IBS and IBS-D correlates with visceral sensitivity ${ }^{6061}$ and symptom severity. ${ }^{62} 63$ However, findings in patients with IBS-C are inconsistent. ${ }^{64} 65$ The expression and levels of the tight junction protein zonula occludens are significantly reduced in IBS-D, and associated with mast cell activation and symptoms. ${ }^{66}$ Confocal laser endomicroscopy studies suggest exposure to certain food antigens can disrupt the epithelial barrier in approximately $50 \%$ of patients with IBS. ${ }^{67}{ }^{68}$ Removal of the reacting antigen from the diet improved symptoms significantly. ${ }^{67}$

\section{The microbiome}

Strong evidence supports a role for bacterial, viral or parasitic infections triggering IBS. ${ }^{51}$ Antibiotic usage may also associate with either the development ${ }^{69}$ or improvement, ${ }^{70}$ of 
IBS symptoms. Moreover, changes in the gut microbiome can modify gastrointestinal motility, visceral sensation, intestinal permeability, stool consistency and visceral sensitivity. ${ }^{71} 72$ In a mouse model, bacterial infection led to an increase in intestinal permeability, which appeared to allow previously tolerated food antigens to activate a localised host immune response in the gastrointestinal tract, via IgE, leading to histamine release, altered motility and visceral hypersensitivity. ${ }^{73}$ There is no conclusive evidence for a specific IBS gut microbiome profile. ${ }^{74} 75$ However, an integrated longitudinal multi-omics analysis of the gut microbiome, metabolome, host epigenome and transcriptome, in the context of host symptoms and physiology in patients with IBS-D and IBS-C, identified subtype-specific and symptom-related variations in microbial composition and function. ${ }^{76}$ It remains unclear whether such microbial changes are secondary and relate to other factors including diet, drugs, altered physiology, including gastrointestinal transit or gastrointestinal water content.

\section{Genetics and epigenetics}

Familial clustering of IBS may be attributed to both genetic and shared environmental factors. ${ }^{778}$ In addition, numerous genetic single nucleotide polymorphisms have been described in association with symptom phenotypes, regulation of neurotransmission, barrier function, inflammatory mediators, ion channels and bile acid metabolism in IBS. ${ }^{77}$ However, a meta-analysis of genes associated with inflammatory mediators found no significant associations for most genes assessed. ${ }^{79}$ There is some evidence for epigenetic changes in IBS, including alterations in DNA methylation, and various miRNAs appear to be associated with increased visceral sensitivity and permeability. ${ }^{80}$

\section{PRESENTATION OF IBS, DIAGNOSIS AND MANAGEMENT IN PRIMARY CARE Overview}

Patients may have IBS-type symptoms for many years without presenting to medical care, often self-managing their symptoms without medical input, and some may never consult. Nevertheless, lower gastrointestinal symptoms frequently prompt people to present to primary care, ${ }^{81}$ accounting for approximately 1 in 12 of all consultations. ${ }^{82}$ Functional gastrointestinal disorders, such as IBS, are by far the most common diagnosis, but symptoms can be difficult to assess and the possibility of colorectal cancer or inflammatory bowel disease (IBD) may create diagnostic uncertainty for clinicians, and anxiety for patients. ${ }^{83}$

General practitioners are the first point of contact and provide the diagnosis and medical care for most people with IBS. Management guidelines encourage general practitioners to make a positive diagnosis of IBS, based on symptoms, in the absence of alarm symptoms or signs that warrant referral to exclude colorectal cancer (box 1), ${ }^{84}$ or abnormalities on simple investigations. ${ }^{10}$ However, persistent abdominal bloating or distension in female patients should prompt consideration of CA-125 and pelvic ultrasound to exclude ovarian cancer. ${ }^{85}$ General practitioners provide the majority of long-term medical care for people with IBS, referring only a minority of patients to specialist clinics. ${ }^{86}$ Compared with hospital settings, general practitioners can, and should, aim to build a long-term relationship with patients, harnessing this to develop a shared understanding of their IBS in the context of their other medical conditions, concerns, priorities and impact on their lives. ${ }^{87} 88$ This can assist in providing
Box 1 Lower gastrointestinal alarm symptoms or signs

that are referral criteria for suspected colorectal cancer ${ }^{84}$

Definite referral criteria

1. Aged $\geq 40$ years with unexplained weight loss and abdominal pain.

2. Aged $\geq 50$ years with unexplained rectal bleeding.

3. Aged $\geq 60$ years with:

a. Iron deficiency anaemia;

b. Change in bowel habit.

4. Positive faecal occult blood test.

Probable referral criteria

1. Adults of any age with an abdominal or rectal mass.

2. Aged $<50$ years with rectal bleeding and any of the following unexplained symptoms or findings:
a. Abdominal pain;
b. Change in bowel habit;
c. Weight loss;
d. Iron deficiency anaemia.

appropriate tailored education, advice and reassurance, shared decision-making and management plans, and be facilitated by an ongoing supportive doctor-patient relationship and prioritisation of continuity of care.

\section{Doctor-patient communication and patient perspectives in primary care}

Establishing an effective doctor-patient relationship and a shared understanding is key to the successful diagnosis and management of chronic conditions such as IBS. ${ }^{89} 90$ Reports from patients with IBS confirm that they would like increased empathy, support and information about the nature of the condition from general practitioners, and options for symptom management. ${ }^{87}$ They often feel their symptoms are dismissed or trivialised, describe the diagnostic process as confusing, or invasive, and the often-lengthy search for efficacious treatments as frustrating. ${ }^{878991-95}$

Patients often seek information and support from multiple sources, including internet web forums, ${ }^{96}$ and may receive conflicting, or incorrect, advice. Clinicians should aim to gain a better understanding of patients' ideas, concerns and expectations of diagnosis and management. Multiple factors influence both the patient's decision to consult their doctor with IBS, and their ability and willingness to self-manage symptoms and engage with treatment. These factors include the impact on their own and their family's lives, social and psychological factors, employment, comorbidities and health beliefs. All should be considered and acknowledged for successful diagnosis and management of IBS in primary care, and good doctor-patient communication generally.

\section{Presentation of IBS to primary care}

General practitioners must assess and manage undifferentiated disease, multiple comorbidities, health anxieties and hidden agendas in brief consultations. Multiple factors, described above, influence patients' decisions to consult. Concerns about serious illness, advice or pressure from friends or relatives, life events and underlying health beliefs can all drive healthcare-seeking behaviour. Understanding reasons for presenting at a particular point in time, especially if symptoms have been present for many years, is important in determining the most appropriate management strategy. 
General practitioners' key skills, especially in relation to chronic disorders such as IBS, are to make a positive diagnosis, including providing a simple explanation of the pathophysiology underlying the symptoms, clarifying the patient's main concerns and managing current symptoms in the wider context of the patient's life. The doctor's relationship with the patient, continuity of care, empathy, including acknowledgement of the impact of symptoms on daily life, a shared understanding of IBS and shared decision-making can assist in providing appropriate education, signposting to reputable online information or peer support, reassurance, advice and management options.

\section{Diagnosis of IBS in primary care}

The key to diagnosis starts with skilled, targeted history taking and examination, considering the patient's medical history and life circumstances. The Rome diagnostic criteria are based on specific symptoms of a defined duration and frequency, ${ }^{2}$ which have been derived predominantly from secondary care patients, and are rarely used in primary care. ${ }^{97}$ Their applicability to clinical practice has been challenged as unnecessarily restrictive, ${ }^{98}$ and only a minority of people diagnosed with IBS in primary care fulfil them. ${ }^{99}$

This restrictive diagnostic approach to IBS may be unhelpful and overly complicated in this setting, where fundamentals of clinical management are common across all these functional gastrointestinal disorders. Applying rigid criteria potentially leaves many patients with troublesome impactful symptoms without a clear diagnosis, increasing uncertainty and leading to issues with providing appropriate advice and management options. The NICE guideline definition of IBS is therefore preferable. ${ }^{10}$

\section{Investigation in primary care}

A positive diagnosis of IBS can be made on the basis of ongoing characteristic symptoms, after assessing for alarm symptoms or signs, and undertaking relevant blood test results, including full blood count (FBC), C reactive protein (CRP) or erythrocyte sedimentation rate (ESR), and serological tests for coeliac disease. ${ }^{10}$ The chance of identifying organic disease on the basis of checking FBC, CRP and ESR in suspected IBS is low, ${ }^{100}$ but the prevalence of abnormal serological testing for coeliac disease is almost three times higher in people with suspected IBS than people without symptoms of IBS, irrespective of predominant stool pattern. ${ }^{101}$ If all these blood tests are normal, other investigations should be minimised. Abdominal and digital rectal examination can help exclude other diagnoses, and may confirm the consistency of stool, including rectal impaction, or identify dyssynergic defaecation (paradoxical contraction on rectal examination during straining) or low rectal masses. ${ }^{102}$ An abdominal X-ray can be considered to rule out faecal loading if constipation is the predominant symptom.

The non-invasive marker of intestinal inflammation faecal calprotectin has enabled risk stratification to prioritise access to investigations to exclude IBD in patients with chronic diarrhoea, reducing unnecessary investigations and referrals from primary to secondary care. ${ }^{103}$ However, calprotectin is not specific to IBD and can be elevated in older age groups (age $\geq 45$ years), obesity, infection, malignancy or by medications, such as proton pump inhibitors (PPIs) or non-steroidal anti-inflammatory drugs (NSAIDs). Local laboratory values vary but, generally, a faecal calprotectin of $<100 \mu \mathrm{g} / \mathrm{g}$ can be considered normal, $100-249 \mu \mathrm{g} / \mathrm{g}$ is borderline and should be repeated, with subsequent referral if persistently elevated, and $\geq 250 \mu \mathrm{g} / \mathrm{g}$ requires urgent referral to secondary care to exclude IBD. ${ }^{103}$ Faecal occult blood or faecal immunochemical testing are not used routinely for assessing patients with possible IBS in primary care, although they are recommended in current guidelines for colorectal cancer screening. ${ }^{104}$ Local and national guidelines for colorectal and ovarian cancer screening should be followed, where indicated. Once a diagnosis of IBS has been made, the general practitioner should endeavour to follow-up the patient within the next 2 months to ensure symptoms are not getting progressively worse, which may be indicative of a more sinister underlying disease process.

\section{When to refer patients to secondary care}

Most patients with IBS are diagnosed and managed by general practitioners in community settings and are never referred to secondary care, even if they have ongoing troublesome symptoms. ${ }^{82}$ In a primary care study of patients with refractory IBS, only $10 \%$ had ever had a secondary care referral. ${ }^{86}$ Reasons for seeking a secondary care opinion include: uncertainty about the diagnosis or alarm symptoms or signs; ongoing refractory symptoms that have not improved despite lifestyle changes (including diet and trials of medication), necessitating initiation of therapies that are unavailable in primary care or patient request for a specialist opinion.

\section{Recommendations}

- Establishing an effective doctor-patient relationship and a shared understanding is key to the management of IBS. Such a relationship can lead to improved quality of life and symptoms, reduce healthcare visits and enhance adherence to treatment (recommendation: strong, quality of evidence: low).

- Patients with IBS would like increased empathy, support and information from clinicians about the nature of the condition, diagnosis and symptom management options (recommendation: strong, quality of evidence: low).

- The National Institute for Health and Care Excellence guideline definition of IBS (abdominal pain or discomfort, in association with altered bowel habit, for at least 6 months, in the absence of alarm symptoms or signs) is more pragmatic and may be more applicable to patients with IBS in primary care than diagnostic criteria derived from patients in secondary care, such as the Rome IV criteria (recommendation: weak, quality of evidence: low).

- All patients presenting with symptoms of IBS for the first time in primary care should have a full blood count, $\mathrm{C}$ reactive protein or erythrocyte sedimentation rate, coeliac serology and, in patients $<45$ years of age with diarrhoea, a faecal calprotectin to exclude inflammatory bowel disease. Local and national guidelines for colorectal and ovarian cancer screening should be followed, where indicated (recommendation: strong, quality of evidence: moderate).

- Clinicians should make a positive diagnosis of IBS based on symptoms, in the absence of alarm symptoms or signs, and abnormalities on simple blood and stool tests (recommendation: strong, quality of evidence: moderate).

- Referral to gastroenterology in secondary care is warranted where there is diagnostic doubt, in patients with symptoms that are severe, or refractory to first-line treatments, or 
where the individual patient requests a specialist opinion (recommendation: weak, quality of evidence: low).

\section{CLINICAL HISTORY AND INVESTIGATION IN SECONDARY CARE}

\section{The first consultation in secondary care}

IBS is one of the most common disorders that gastroenterologists deal with, accounting for at least $10 \%$ of consultations in the outpatient clinic. ${ }^{105}$ Patients presenting to secondary care often attend the consultation with the hope that the specialist can diagnose an organic disease that has been missed in primary care. This may be because a diagnosis of IBS is stigmatised, ${ }^{106}$ or considered, erroneously, as a purely psychological disorder, ${ }^{107}$ or due to unexpressed concerns of a missed sinister cause of their symptoms. These aspects should all be considered by the secondary care clinician when managing patient expectations and selecting appropriate investigations. ${ }^{107}$ It is equally important to build rapport and to build trust in the doctor-patient relationship in secondary care by adopting the principles of empathic listening to optimise the interaction. ${ }^{108}$ Evidence suggests that 2 minutes of active listening at the beginning of a consultation gives the patient the feeling of having being listened to, ${ }^{109}$ and therefore having confidence in subsequent decisions around their care. An empathic approach can improve quality of life and symptoms, ${ }^{110}$ reduce healthcare visits and enhance adherence to treatment. ${ }^{108} 111$ The principles of history taking are similar to those in primary care. It is useful to screen for potential symptom triggers, including previous acute enteric infection, present in approximately $10 \%$ of people with IBS, ${ }^{112}$ antibiotics or psychological stress. This demonstrates to the patient that the clinician is interested in understanding their disorder, and helps the patient's understanding of the possible underlying aetiology and validates the diagnosis.

It is important to start the consultation by asking when the patient's symptoms started (figure 1). A detailed history should confirm presence of the cardinal symptoms of IBS. These include abdominal pain and altered bowel habit (abnormal stool frequency and/or consistency) and, in particular, the relationship between the two, remembering that the location of pain can be in the upper or lower abdomen. Importantly, the clinician needs to assess whether the patient recognises that there is a link between the pain and the alteration in bowel habit. Thus, pain can be relieved or exacerbated by defaecation, or associate temporally with changes in bowel habit (eg, the pain is present when the individual is more constipated or has worsening diarrhoea). Predominant stool pattern, on days when the patient's stools are abnormal, should be assessed using the Bristol stool chart. ${ }^{113}$ Attention should also be paid to other gastrointestinal symptoms. The presence of bloating is not required to fulfil the Rome IV criteria but, if present, is highly suggestive of IBS, and is often accompanied by visible abdominal distension. ${ }^{114}$ Although the Rome IV criteria are the gold standard to define IBS for research purposes, they are probably overly restrictive for use, even in secondary care, and a pragmatic definition in line with

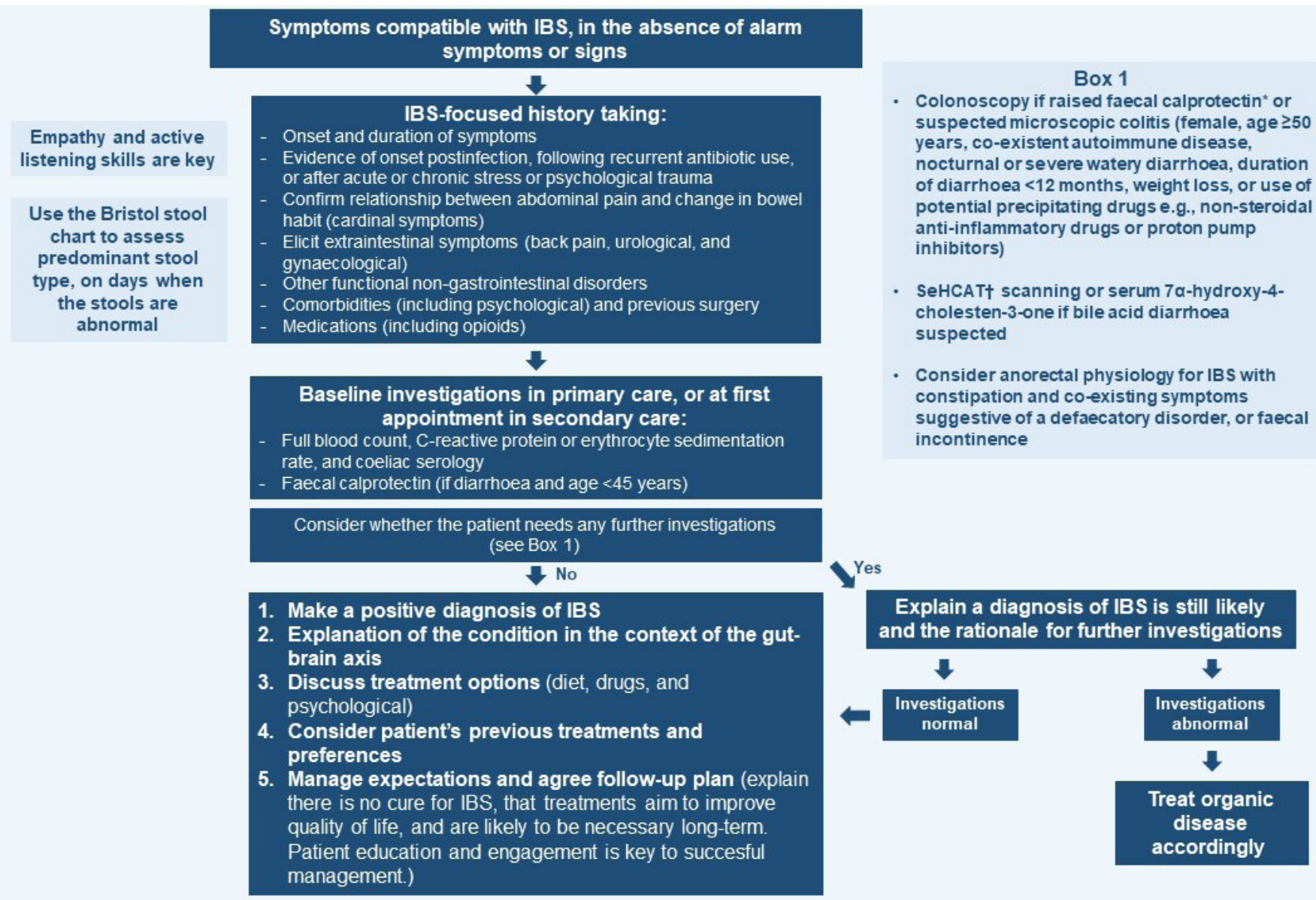

Figure 1 Diagnostic algorithm detailing the approach to the positive diagnosis of IBS. *If the initial faecal calprotectin level is abnormal (eg, $\geq 250$ $\mu \mathrm{g} / \mathrm{g}$ ), the suspicion for IBD is high, proceed to colonoscopy; if the initial faecal calprotectin level is indeterminate according to local laboratory values $(\mathrm{eg}, 100-249 \mu \mathrm{g} / \mathrm{g})$, repeat the test off non-steroidal anti-inflammatory drugs, proton pump inhibitors, etc, and refer for colonoscopy if the repeat test remains indeterminate or is abnormal. TSeHCAT, 23-seleno-25-homotaurocholic acid. 
that used in the NICE guideline, ${ }^{10}$ and outlined above, should be preferred.

Coexistent early satiety, postprandial fullness, epigastric pain, nausea or heartburn are common, as functional dyspepsia and gastro-oesophageal reflux frequently overlap with IBS. $^{115-117}$ Extraintestinal symptoms, such as back pain, bladder and gynaecological symptoms, and insomnia are frequent, as is the presence of other functional somatic disorders, such as fibromyalgia, tension headache or chronic fatigue. ${ }^{118}$ Common mental disorders, and somatoform-type behaviour, often coexist. ${ }^{119} 120$ A patient with more severe IBS may volunteer a history of abuse, or respond to a cue when told that 'some people report abuse as a possible cause...'. It is, therefore, important to consider all these factors when assessing a patient with a possible diagnosis of IBS during the initial consultation, as they add diagnostic value, and predict the degree of functional limitation of the condition, reduction in quality of life and healthcare utilisation. ${ }^{117}$ This may prevent presentation to multiple other specialities, and avoid iatrogenic harm from unnecessary interventions. ${ }^{121}$ Objective evidence of weight loss is also important to assess and document. Other relevant items in the clinical history include previous surgical interventions, and a family history of gastrointestinal cancer, IBD, coeliac disease or IBS. Finally, attention should be taken to exclude gastrointestinal symptoms related to a change in diet, drugs that can alter gut motility, such as psychotropic agents or opioids or alcohol excess.

\section{Investigations in secondary care}

In a patient with normal investigations from primary care on referral, exhibiting typical symptoms, and in the absence of alarm symptoms or signs, or atypical features, the diagnosis of IBS is secure (figure 1). A validation study of the Rome IV criteria in secondary care demonstrated this was particularly the case for IBS-C and IBS$\mathrm{M}^{21}$ Patients meeting these criteria were 21 times more likely to have IBS-C than to not have IBS-C, and 11 times more likely to have IBS-M than to not have IBS-M after limited diagnostic workup. The clinician should, therefore, appear confident and, after clinical assessment is complete, communicate a positive diagnosis of IBS based on symptoms. In those with alarm symptoms or signs, urgent referral for colonoscopy or radiological evaluation of the colon is required, ${ }^{10}$ although the diagnostic performance of alarm symptoms or signs is modest, ${ }^{122}$ and up to $80 \%$ of patients with IBS in primary and secondary care will report at least one alarm symptom. ${ }^{123}$ In those with atypical features, such as nocturnal diarrhoea or abdominal pain, or features of obstructive defaecation, further limited investigation may be required to exclude important mimics. ${ }^{124}$ These include microscopic colitis or primary, or idiopathic, bile acid diarrhoea (BAD) in those with suspected IBS-D, and dyssynergic defaecation and other defaecatory disorders in those with suspected IBS-C.

The yield of colonoscopy in patients with IBS is extremely low, ${ }^{125}$ and there is no evidence of reassurance being derived by patients from a normal examination. ${ }^{126}$ However, colonoscopy to exclude microscopic colitis should be considered in patients with diarrhoea. Factors that should alert the clinician to the possibility of microscopic colitis include female sex, age $\geq 50$ years, coexistent autoimmune disease, nocturnal or severe, watery, diarrhoea, duration of diarrhoea $<12$ months, weight loss or use of potential precipitating drugs including NSAIDs, PPIs, selective serotonin reuptake inhibitors (SSRIs) or statins. ${ }^{127-129}$

In primary $\mathrm{BAD}$, bile acids enter the colon, enhancing mucosal permeability, inducing water and electrolyte secretion, and accelerating colonic transit. The condition is diagnosed via 23-seleno-25-homotaurocholic acid (SeHCAT) scanning, although this may be unavailable in some countries. ${ }^{130}$ A serum $7 \alpha$-hydroxy-4-cholesten-3-one is a reasonable alternative. The current BSG guideline for the investigation of chronic diarrhoea does not recommend a therapeutic trial of a bile acid sequestrant as a diagnostic test for $\mathrm{BAD},{ }^{131}$ because a lack of response does not exclude the condition. ${ }^{132}$ Symptoms of BAD can mimic IBS-D, with between one-in-three and one-in-four patients with suspected IBS-D having an abnormal SeHCAT retention, ${ }^{133} 134$ and response rates to a bile acid sequestrant are higher at retentions of $<10 \%$ or $<5 \% .^{135}$ Predictors of primary BAD are lacking, other than higher body mass index, ${ }^{133}$ but if nocturnal or severe diarrhoea is present the diagnosis should be considered. BAD should also be suspected in patients with symptoms suggestive of IBS-D with prior cholecystectomy.

Symptoms suggestive of a defaecatory disorder include straining at stool, a sensation of incomplete, or blocked, evacuation and use of digital manoeuvres to facilitate defaecation. However, these symptoms are common in patients with IBS-C, as well as in those with functional constipation, and whether they arise due to different pathophysiological mechanisms in the two disorders is unclear. ${ }^{136} 137$ In the presence of these symptoms, or of faecal incontinence, physiological testing could be considered, where available, to facilitate selection of patients most likely to benefit from targeted pelvic floor biofeedback therapy to improve anorectal function. ${ }^{138}$ Particular caution should be given to considering surgical correction of anorectal anatomic alterations in patients with typical symptoms of IBS-C, as no prospective studies have demonstrated surgery improves symptoms. ${ }^{139}$ Likewise, abdominal pain is considered a relative contraindication to surgical correction of refractory slow transit constipation. $^{139}$

Some investigators have reported a high prevalence of exocrine pancreatic insufficiency (EPI) in patients with suspected IBS, ${ }^{140}$ although other studies have not confirmed this. ${ }^{141}$ The current BSG guideline for the investigation of chronic diarrhoea only recommends screening patients for EPI, via faecal elastase, if features consistent with fat malabsorption are present. ${ }^{131}$ Similarly, testing patients with suspected IBS-D for EPI is not recommended, unless steatorrhoea is reported. Finally, there is no role for hydrogen breath testing to exclude lactose intolerance or small intestinal bacterial overgrowth in patients with IBS, particularly as these tests may be falsely positive in patients with IBS, due to rapid transit. ${ }^{142}$ Studies using small intestinal aspiration, considered the gold standard for diagnosing small intestinal bacterial overgrowth, have not demonstrated an increased prevalence of the condition in suspected IBS, ${ }^{143}$ and the lactulose breath test correlates poorly with small intestinal aspiration. ${ }^{144}$ In addition, despite this being the rationale for use of nonabsorbable antibiotics in IBS, ${ }^{70}$ a positive breath test result does not predict response to treatment. ${ }^{145}$ Similarly, variants in the sucrase-isomaltase gene have been reported to be associated with an increased risk of IBS, ${ }^{146}$ and some investigators have reported evidence of sucrase-isomaltase deficiency on small intestinal biopsy in patients with suspected IBS, ${ }^{147}$ but at present there is insufficient evidence for consideration of routine testing.

\section{Communicating a positive diagnosis and management plan in secondary care}

A diagnosis of IBS needs to be communicated clearly to the patient using simple words and explanations. There is evidence that patient education about the condition can lead to an improvement in symptoms. ${ }^{148}$ It should be underlined that IBS is a chronic disorder, with recurrent fluctuating symptoms 
triggered by stress, intercurrent illnesses, drugs and often the act of eating. IBS is not associated with an increased risk of cancer or mortality, ${ }^{149}$ but affects quality of life to the same degree as organic gastrointestinal diseases, such as IBD. ${ }^{13}$ The main pathophysiological aspect is related to visceral hypersensitivity, which is also the principal target of many current treatments. Therefore, explaining IBS as a disorder of gut-brain interaction, together with a simple account of the gut-brain axis and how this is impacted by diet, stress, cognitive, behavioural and emotional responses to symptoms and postinfective changes is important. Such an approach may improve patient understanding and acceptance of a diagnosis of IBS, and engagement with a shared management plan to include an explanation of the mechanisms of action, potential side effects and rationale for the use of drugs or psychological and dietary therapies within the context of the gut-brain axis.

\section{Recommendations}

- There is no role for colonoscopy in IBS, other than in those with alarm symptoms or signs, or those with symptoms suggestive of IBS with diarrhoea who have atypical features and/or relevant risk factors that increase the likelihood of them having microscopic colitis (female sex, age $\geq 50$ years, coexistent autoimmune disease, nocturnal or severe, watery, diarrhoea, duration of diarrhoea $<12$ months, weight loss or use of potential precipitating drugs including non-steroidal anti-inflammatory drugs, proton pump inhibitors, etc) (recommendation: strong, quality of evidence: moderate).

- In those with symptoms suggestive of IBS with diarrhoea, but with atypical features such as nocturnal diarrhoea, or a prior cholecystectomy, 23-seleno-25-homotaurocholic acid scanning or serum $7 \alpha$-hydroxy-4-cholesten-3-one should be considered to exclude bile acid diarrhoea (recommendation: strong, quality of evidence: low).

- In patients with IBS and coexisting symptoms suggestive of a defaecatory disorder or faecal incontinence, anorectal physiology tests can be considered, where available, to select those who might benefit from biofeedback (recommendation: weak, quality of evidence: low).

- There is no role for testing for exocrine pancreatic insufficiency, or for hydrogen breath testing to rule out small intestinal bacterial overgrowth or carbohydrate intolerance, in patients with typical IBS symptoms (recommendation: strong, quality of evidence: weak).

- The diagnosis of IBS, its underlying pathophysiology and the natural history of the condition, including common symptom triggers, should be explained to the patient. This should introduce the concept of IBS as a disorder of gutbrain interaction, together with a simple account of the gutbrain axis and how this is impacted by diet, stress, cognitive, behavioural and emotional responses to symptoms, and postinfective changes (recommendation: strong, quality of evidence: weak).

\section{TREATMENT OF IBS}

\section{General overview}

The treatment of IBS is generally directed towards the predominant symptom, or symptoms, experienced by the patient. All patients should be advised of the potential benefits of regular exercise, as there is some evidence from RCTs that this can be beneficial, ${ }^{150151}$ particularly for constipation, ${ }^{150}$ with beneficial effects still apparent at 5 years in one trial. ${ }^{152}$ Otherwise, treatment should commence with dietary therapies or first-line drugs, according to patient choice, with second-line drugs reserved for those whose symptoms do not improve with these measures, due to a combination of the potential side effects, as well as the costs, of some of these agents to the health service. Most second-line drugs are only available in secondary care. Ideally, the efficacy of selected treatments should be reviewed at 3 months, and discontinued if no response, with escalation to the next available therapy (figure 2). Currently, psychological therapies are reserved for patients whose symptoms are refractory to drugs, although more research is required to explore the efficacy of earlier use, and it may be worth mentioning them earlier on so that patients have the option to consider them, and so that they are not viewed as a last resort. There should be a realistic discussion concerning the limitations of all available treatments for IBS to manage expectations. It is important to stress that cure is unlikely, but substantial improvement in symptoms, social functioning and quality of life is achievable. The final decision regarding treatment choices should be made by the patient, with advice and support from the clinician.

\section{Recommendations}

- All patients with IBS should be advised to take regular exercise (recommendation: strong, quality of evidence: weak).

\section{Methodology for systematic reviews of IBS therapy}

To inform this guideline, we updated a series of systematic reviews and trial-based or network meta-analyses conducted by some of the authors. ${ }^{153-163}$ The aim was to assess the efficacy of dietary modifications and therapies, unlicensed, as well as licensed, pharmacological therapies, and psychological therapies in IBS. We considered RCTs comparing pharmacological therapies with placebo, psychological therapies with either no treatment or standard/usual care or dietary therapies with standard dietary advice, habitual diet or a sham dietary therapy. Crossover trials were eligible for inclusion, provided extractable data were available at the end of the first treatment period, prior to cross-over. Studies recruited adults from primary, secondary or tertiary care with IBS symptoms diagnosed by any criteria (including clinical impression). Trials had to assess the effect of treatment in terms of either improvement of IBS symptoms, or improvement of abdominal pain, as a dichotomous assessment. It is important to point out that most RCTs of first-line treatments, as well as gut-brain neuromodulators and psychological therapies, used less rigorous end points to judge treatment efficacy, such as improvement in, or satisfactory relief of, global symptoms or abdominal pain. Trials of novel second-line drugs, on the other hand, tend to use Food and Drug Administration (FDA)approved end points to judge efficacy, consisting of a $\geq 30 \%$ improvement in abdominal pain, an increase in the number of complete spontaneous bowel movements (CSBMs) per week in IBS-C, or a reduction in the number of days with stools of loose consistency in IBS-D, and composites thereof.

We considered the following treatments: soluble or insoluble fibre, a diet low in fermentable oligosaccharides, disaccharides and monosaccharides and polyols (FODMAPs), a gluten-free diet, probiotics, antidiarrhoeals, antispasmodic drugs (including peppermint oil), laxatives, gut-brain neuromodulators (tricyclic antidepressants (TCAs) and SSRIs, previously termed antidepressant drugs), eluxadoline, 5- $\mathrm{HT}_{3}$ receptor antagonists, antibiotics, secretagogues, $5-\mathrm{HT}_{4}$ receptor agonists or psychological therapies (including gut-directed hypnotherapy) (online supplemental table 1). As this was an update of prior meta-analyses, ${ }^{153-163}$ we searched MEDLINE, EMBASE, EMBASE Classic and the 


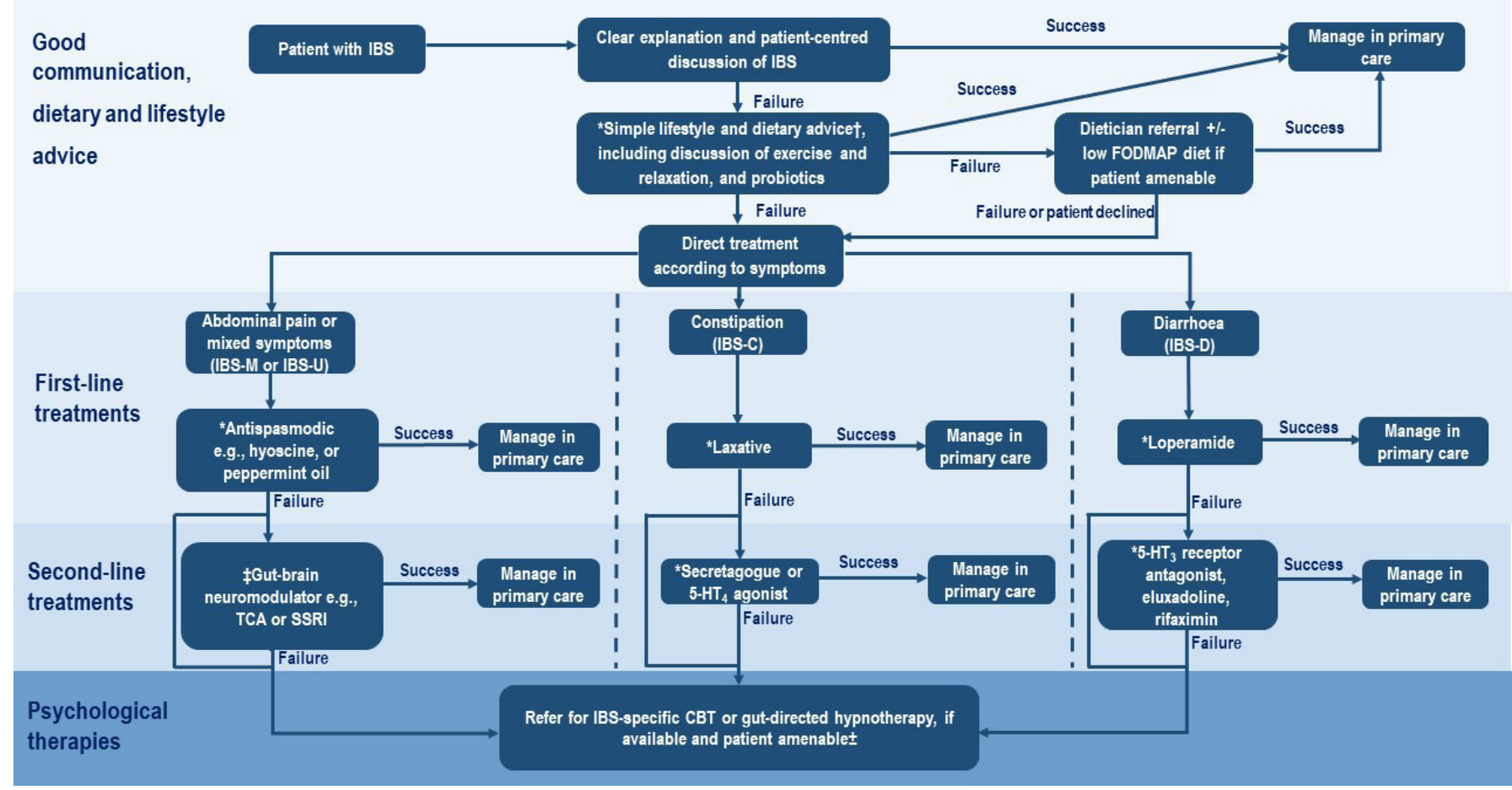

Figure 2 Treatment algorithm for IBS. * Review efficacy after 3 months of treatment and discontinue if no response. †As per the National Institute for Health and Care Excellence IBS dietary advice sheet, plus consider ispaghula. ¥TCAs should be first choice, starting at a dose of 10 $\mathrm{mg}$ at night, and titrating slowly (eg, by $10 \mathrm{mg} /$ week) according to response and tolerability. Continue for at least 6 months if the patient reports symptomatic response. \pm Where available locally, and based on patient preference, psychological therapies can be considered at an earlier stage, but are recommended strongly when symptoms are refractory to drug treatment for 12 months. 5-HT, 5-hydroxytryptamine; CBT, cognitive behavioural therapy; FODMAP, fermentable oligosaccharides, disaccharides and monosaccharides and polyols; IBS-C, IBS with constipation; IBS-D, IBS with diarrhoea; IBS-M, IBS with mixed bowel habits; IBS-U, IBS unclassified; SSRI, selective serotonin reuptake inhibitor; TCA, tricyclic antidepressant.

Cochrane central register of controlled trials between January 2017 and September 2020. The search strategy is provided in the online supplemental file 1 . No restrictions were applied regarding language of publication. We conducted a recursive search of the bibliography of eligible articles. The lead reviewer (ACF) screened titles and trial abstracts that had been identified by the search strategy for articles that could possibly be eligible for the review. The lead reviewer (ACF) then screened the selected trials to confirm eligibility, using predesigned eligibility forms. A second reviewer (CJB), masked to the initial assessment, also evaluated all identified trials for eligibility. We resolved discrepancies by discussion, with a consensus view taken, and used the kappa statistic to measure the degree of agreement for judging study eligibility.

The literature search identified 4111 citations, of which 46 appeared to be relevant, and 17 were eligible and were incorporated into this guideline. ${ }^{164-180}$ Fourteen of these were used to update meta-analyses. ${ }^{164-177}$ Agreement between reviewers for study eligibility was excellent (kappa statistic $=0.81$ ). Of these 14 studies, 2 compared linaclotide with placebo, ${ }^{164} 165$ and were used to update a previous network meta-analysis, ${ }^{155} 8$ compared various probiotics with placebo, ${ }^{166-173}$ and were used to update an existing trial-based meta-analysis ${ }^{158}$ and 4 were RCTs of a low FODMAP diet, ${ }^{174-177}$ and again were used to update a prior trial-based meta-analysis. ${ }^{161}$ The remaining 3 RCTs were an 8 -week trial of bimodal release ondansetron in IBS-D, ${ }^{178}$ and 2 phase II trials of minesapride ${ }^{179} 180$ a novel 5- $\mathrm{HT}_{4}$ receptor agonist. The results of these latter 3 trials are discussed briefly below. Recommendations for all other treatments are, therefore, made based on the results of existing trial-based and network meta-analyses.

All data for newly identified RCTs were extracted independently by two investigators (ACF and CJB) on to a Microsoft Excel spreadsheet (XP professional edition; Microsoft, Redmond, Washington, USA). We resolved disagreements between investigators by discussion. We extracted data as intention-to-treat analyses, with all dropouts assumed to be treatment failures, wherever trial reporting allowed this. We incorporated data from newly identified trials into existing trial-based and network meta-analyses. As we examined binary outcomes (global IBS symptoms or abdominal pain improved or not improved), we expressed the impact of each intervention as a relative risk (RR) of global IBS symptoms or abdominal pain not improving, together with $95 \%$ CIs, where if the RR is $<1$ and the 95\% CI does not cross 1 , there is a significant benefit of the intervention over the control. This approach is the most stable, compared with RR of improvement, or using the OR, for some meta-analyses. ${ }^{181}$

We used Review Manager V.5.4.1 (RevMan for Windows 2020, the Nordic Cochrane Centre, Copenhagen, Denmark) for updates to trial-based meta-analyses. We conducted updated network meta-analyses using the frequentist model, with the statistical package 'netmeta' (V.0.9-0, https://cran.r-project. org/web/packages/netmeta/index.html) in R (V.4.0.2). Network meta-analysis usually gives a more precise estimate, compared with results from standard, trial-based meta-analysis. ${ }^{182} 183$ It can 
also rank treatments to inform clinical decisions, ${ }^{184}$ according to their P-score, which is a value between 0 and 1 , with higher scores indicating a greater probability of a treatment being ranked as best. ${ }^{185}$ For both trial-based and network metaanalyses, we pooled data using a random effects model, to give a more conservative estimate of the efficacy of individual therapies, ${ }^{186}$ and assessed heterogeneity using the $\mathrm{I}^{2}$ statistic, which ranges from $0 \%$ to $100 \%$, with $0 \%$ representing no observed heterogeneity, and larger values indicating increasing heterogeneity. A value $\leq 50 \%$ was chosen to represent low levels of heterogeneity. ${ }^{187}$

\section{Fibre and dietary therapies}

Over $80 \%$ of individuals with IBS report food-related symptoms, especially to fermentable carbohydrates and fats. ${ }^{188} 189$ Patients reporting adverse food reactions experience more severe gastrointestinal symptoms, associated subjective health complaints of musculoskeletal pains and chronic fatigue and reduced quality of life, compared with those without food sensitivities. ${ }^{189-191}$ Hence, most patients with IBS are keen to explore dietary options, with over $60 \%$ wanting to know what food(s) they should avoid, and up to $70 \%$ having modified their diet. ${ }^{192}$ There are multiple mechanisms by which food may trigger symptoms in IBS, including primary effects (eg, osmotic, chemical, immunological, mechanical or neuroendocrine) and secondary effects (eg, fermentation by-products, alterations in intraluminal $\mathrm{pH}$ or effects on the gut microbiome). ${ }^{193} 194$

Patients may seek to undertake dietary manipulations based on tests that suggest potential food intolerances. A prior RCT, comprising 150 patients with IBS and positive $\operatorname{IgG}$ antibodies to food, found a significant improvement in symptoms in those allocated to a true-exclusion diet, compared with a shamexclusion diet. ${ }^{195}$ However, the effect was modest and there are concerns regarding the poor specificity and applicability of IgG antibody testing. ${ }^{196}$ For example, IgG antibodies to yeast were reported in $87 \%$ of patients yet are rarely responsible for symptoms following dietary rechallenges. ${ }^{195} 196$ Hence, food elimination diets based on IgG antibodies are not recommended. Although some studies have identified potential food intolerances via leucocyte antigen testing of peripheral blood samples or real-time confocal laser endomicroscopy, this requires further corroboration. $^{68197}$

In clinical practice, the last decade has seen a growing interest in the use of three diets for IBS, which are traditional dietary advice, a low FODMAP diet or a gluten-free diet. Of these, traditional dietary advice is considered as first-line, and is based on guidance produced by NICE and the British Dietetic Association (BDA). ${ }^{198} 199$ Its principles, which do not require formal dietetic input, include adopting healthy eating patterns, such as regular meals, maintaining adequate nutrition, limiting alcohol and caffeine intake, adjusting fibre intake, and reducing consumption of fatty and spicy foods. However, the evidence for this is based on a combination of clinical experience and the potential mechanisms by which these foods may induce gastrointestinal symptoms in IBS, rather than evidence from RCTs of this approach versus a control treatment. With regard to fibre, a systematic review and meta-analysis of 15 RCTs, comprising 946 patients, demonstrated its benefit in IBS (RR of symptoms persisting $=0.87 ; 95 \%$ CI 0.80 to 0.94 ) (online supplemental figure 1). ${ }^{158}$ However, this effect was limited to soluble fibre, such as ispaghula (RR $0.83 ; 95 \%$ CI 0.73 to 0.94 ), but not insoluble fibre, like wheat bran, which may exacerbate abdominal pain and bloating. It is generally advised to start with low doses of soluble fibre (3-4 g daily) and build up gradually, as tolerated, to a total dosage of $20-30 \mathrm{~g} /$ day, as it increases colonic water content and volume, ${ }^{200}$ which may aggravate abdominal pain and bloating.

A low FODMAP diet is recommended as a second-line diet for IBS. ${ }^{10198}$ FODMAPS are short-chain fermentable carbohydrates that are found in a variety of fruits, vegetables, dairy products, artificial sweeteners and wheat. They increase small intestinal water volume and colonic gas production and, in those with visceral hypersensitivity, induce gastrointestinal symptoms. ${ }^{201}$ They may also trigger bowel symptoms as they produce short chain fatty acids, which lower colonic $\mathrm{pH} .{ }^{202}$ Hence, the benefits of adopting a low FODMAP diet in IBS seem physiologically plausible and several RCTs have evaluated its efficacy in IBS. The update to the prior systematic review and meta-analysis, ${ }^{161}$ used to inform this guideline, identified 11 trials comparing a low FODMAP diet with various dietary control interventions, including habitual diet, a high FODMAP diet, traditional dietary advice as recommended by NICE and the BDA or a sham diet, in 658 participants. A low FODMAP diet was associated with a reduction in the risk of remaining symptomatic, compared with all control interventions (RR $0.71 ; 95 \%$ CI 0.61 to 0.83 ) (online supplemental figure 2). However, as reported in the prior meta-analysis, ${ }^{161}$ the quality of evidence from these trials was very low, due to small sample sizes, difficulties in blinding and heterogeneity between studies. The latter has mainly been attributed to the various control interventions used. Interestingly, those studies that compared the low FODMAP diet with traditional dietary advice from NICE and the BDA had the least heterogeneity, but also the least magnitude of effect (RR 0.82; $95 \%$ CI 0.67 to 1.01 ), suggesting that the $50 \%-70 \%$ symptomatic benefit reported for a low FODMAP diet in some studies may have been overestimated. Moreover, RCTs have focused solely on the initial 'elimination' phase of the low FODMAP diet, which lasts between 4 and 6 weeks, not the subsequent reintroduction and long-term 'personalisation' phase. The effect of FODMAP reintroduction to tolerance on IBS symptoms is therefore unclear, although there have been open-label studies reporting the long-term efficacy of an adapted low FODMAP diet ranges somewhere between 50\% and 60\%. ${ }^{177} 203$

Finally, some patients with IBS report symptomatic benefit from a gluten-free diet despite no objective evidence of coeliac disease. ${ }^{204}$ The prior systematic review and meta-analysis identified only $2 \mathrm{RCTs},{ }^{161}$ comprising 111 participants and noted that although a gluten-free diet was associated with a reduction in global symptoms compared with a control diet, this was not statistically significant (RR $0.42 ; 95 \%$ CI 0.11 to 1.55 ). There is, therefore, insufficient evidence to recommend a gluten-free diet routinely in IBS, although given its widespread popularity further RCTs are needed. It has also been suggested that the clinical benefit reported with a gluten-free diet is, in the main, not due to the removal of gluten per se, but rather a reduction in dietary fructan content, which is a FODMAP, resulting from wheat exclusion. ${ }^{205} 206$ Future trials comparing a gluten-free diet head-to-head with a low FODMAP diet with regard to efficacy, convenience, cost and acceptability, may better inform patient choice.

Issues with following a low FODMAP diet, as opposed to traditional dietary advice, include the need for a specialist dietitian to implement it, followed by close monitoring to avoid nutritional deficiencies or the development of overly restrictive eating habits. ${ }^{207-209}$ The latter emphasises the importance of screening out patients at high risk for such behaviour prior to recommending such diets. ${ }^{207-209}$ This can be achieved using 
simple eating disorder questionnaires (eg, SCOFF), applying these carefully and with empathy, to identify those with high levels of psychological distress. ${ }^{210}{ }^{211}$ Moreover, a low FODMAP diet may induce detrimental changes to the gut microbiota, with reductions in Bifidobacteria and total bacterial count, ${ }^{212}$ although the long-term consequences of this are unknown. Future studies should aim to identify actionable biomarkers that might predict response to a given dietary intervention. Preliminary data suggest that response to a low FODMAP diet may be predicted from baseline faecal bacterial profile and metabolomic activity, while a gluten-free diet may benefit those with serum antigliadin antibodies. ${ }^{213-215}$

\section{Recommendations}

- First-line dietary advice should be offered to all patients with IBS (recommendation: strong, quality of evidence: weak).

- Food elimination diets based on IgG antibodies are not recommended in patients with IBS (recommendation: strong, quality of evidence: moderate).

- Soluble fibre, such as ispaghula, is an effective treatment for global symptoms and abdominal pain in IBS, but insoluble fibre (eg, wheat bran) should be avoided as it may exacerbate symptoms. Soluble fibre should be commenced at a low dose (3-4 g/day) and built up gradually to avoid bloating (recommendation: strong; quality of evidence: moderate).

- A diet low in fermentable oligosaccharides, disaccharides and monosaccharides and polyols, as a second-line dietary therapy, is an effective treatment for global symptoms and abdominal pain in IBS, but its implementation should be supervised by a trained dietitian and fermentable oligosaccharides, disaccharides and monosaccharides and polyols should be reintroduced according to tolerance (recommendation: weak, quality of evidence very low).

- A gluten-free diet is not recommended in IBS (recommendation: weak, quality of evidence very low).

\section{Probiotics}

The faecal microbiome of patients with IBS may differ significantly from that of healthy individuals. ${ }^{216}$ The theory that this might, in part, be involved in pathophysiology has led to interest in whether probiotics, which are live or attenuated microorganisms that may have beneficial effects in humans, can be used to alter the microbiome, improving symptoms. We updated a prior meta-analysis of $37 \mathrm{RCTs},{ }^{158}$ incorporating data from 8 new trials ${ }^{166-173}$ and randomising 6352 patients. Subgroup analyses according to type of probiotic used (where more than one trial of a particular group of probiotics was conducted) demonstrated significant effects on global symptoms or abdominal pain for combinations of probiotics (RR $0.79 ; 95 \%$ CI 0.70 to 0.89 ), Lactobacillus (RR $0.75 ; 95 \%$ CI 0.60 to 0.94 ), Bifidobacterium (RR 0.80; 95\% CI 0.70 to 0.91) and Escherichia (RR 0.86; 95\% CI 0.79 to 0.93 ) (online supplemental figure 3). Adverse event rates were similar in the probiotic and placebo arms. Variations in study design, strain and species of probiotic used, and heterogeneity between studies make it difficult to give specific recommendations. However, it is reasonable to advise patients wishing to try probiotics to take them for up to 12 weeks, and to discontinue treatment if there is no improvement in symptoms.

\section{Recommendations}

- Probiotics, as a group, may be an effective treatment for global symptoms and abdominal pain in IBS, but it is not possible to recommend a specific species or strain. It is reasonable to advise patients wishing to try probiotics to take them for up to 12 weeks, and to discontinue them if there is no improvement in symptoms (recommendation: weak, quality of evidence: very low).

\section{Drugs used first line for IBS}

Loperamide is a synthetic $\mu$-opioid agonist that reduces myenteric plexus activity, thereby increasing intestinal transit time and enhancing water reabsorption. A prior systematic review identified only 2 RCTs of loperamide in IBS-D and IBS-M containing 42 patients. ${ }^{160}$ Although the drug improved stool frequency and consistency, it had no effect on global symptoms (RR $0.44 ; 95 \%$ CI 0.14 to 1.42 ). The incidence of adverse events with loperamide was similar to placebo in these trials. However, in clinical practice, abdominal pain, bloating, nausea and constipation are common side effects, and may limit tolerability. Titrating the dose carefully may improve tolerability.

Antispasmodics are among the most frequently used over-thecounter treatments for IBS, and can be divided, broadly, into antimuscarinics and smooth muscle relaxants. Antimuscarinics, including dicycloverine, propantheline, otilonium bromide and hyoscine butylbromide reduce intestinal motility, whereas alverine and mebeverine are direct-acting intestinal smooth muscle relaxants. The proposed mechanism of action of these agents is based on the assumption that some IBS symptoms are a result of gastrointestinal spasm and dysmotility, which antispasmodics ameliorate ${ }^{217} \mathrm{~A}$ prior meta-analysis identified $26 \mathrm{RCTs}$, containing 2811 patients, which compared 13 different antispasmodics with placebo. ${ }^{160}$ Despite significant heterogeneity between trials, presumably driven by differences in antispasmodics studied, patient selection and study design, fewer patients treated with antispasmodics had persistent global symptoms or abdominal pain (RR 0.65 ; 95\% CI 0.56 to 0.76 ) (online supplemental figure 4). However, these findings should be interpreted with caution, given heterogeneity between trial results and the varying end points studied. In addition, most trials recruited unselected patients with IBS, so whether the proposed reduction in gastrointestinal motility with antispasmodics improves diarrhoea is unclear. Access to some of these drugs is limited, although hyoscine butylbromide is available widely; pooled results from 3 RCTs, containing 426 patients, demonstrated efficacy (RR 0.63 ; 95\% CI 0.51 to 0.78 ) (online supplemental figure 4). ${ }^{160}$ In contrast, in this meta-analysis, neither alverine nor mebeverine demonstrated benefit over placebo. The overall rates of adverse events were significantly higher with antispasmodics compared with placebo; most notably dry mouth, visual disturbance and dizziness. ${ }^{160}$

Peppermint oil is another popular over-the-counter remedy for IBS. Although not completely understood, its putative antispasmodic action is via L-menthol's blockade of calcium channels. ${ }^{218}$ A meta-analysis of 8 RCTs, which included 823 patients, has evaluated its efficacy. ${ }^{162}$ For global symptoms or abdominal pain, peppermint oil was more efficacious than placebo (RR 0.58; $95 \%$ CI 0.34 to 0.98 ) (online supplemental figure 5). It should be stressed that these trials involved specific formulations of peppermint oil. Their results, therefore, cannot be extrapolated to other formulations and, in the largest trial to date included in this meta-analysis, which used two formulations of variable release peppermint oil (small bowel vs ileocolonic) there was no benefit over placebo for the primary end point. ${ }^{219}$ In addition, low study quality, the lack of consistent use of the Rome criteria to define IBS and heterogeneity between RCTs limit confidence in the data. There is also a lack of information as to which 
IBS subtype would benefit most. Overall, adverse events with peppermint oil were no more common than placebo, ${ }^{160}$ although patients can report gastro-oesophageal reflux symptoms due to its effects on the lower oesophageal sphincter. Comparison of peppermint oil and antispasmodics with other unlicensed or 'traditional' treatments for IBS, including ispaghula and gutbrain neuromodulators, in a network meta-analysis suggested that, for global symptoms, peppermint oil was ranked first and antispasmodics third, with both superior to placebo (online supplemental figure 6). ${ }^{157}$ In terms of effect on abdominal pain, antispasmodics ranked second, with peppermint oil third (online supplemental figure 7). Again, both were significantly more efficacious than placebo.

Current NICE guidance for the management of IBS suggests that patients with IBS-C can be treated with laxatives, advising dose titration according to symptoms. ${ }^{10}$ Although both stimulant and osmotic laxatives are efficacious in the treatment of chronic idiopathic constipation, ${ }^{220}$ only the latter have been evaluated in 2 RCTs of polyethylene glycol, recruiting 181 patients with IBS-C. ${ }^{221} 222$ In one trial, there was no significant effect on either abdominal pain or number of bowel movements, ${ }^{221}$ and in the second the number of bowel movements increased significantly, but with no improvement in abdominal pain. ${ }^{222}$ Polyethylene glycol was generally well-tolerated with abdominal pain the most frequent adverse event. The long-term efficacy of osmotic laxatives in IBS-C is unknown, as both trials were of only 4 weeks duration.

\section{Recommendations}

- Loperamide may be an effective treatment for diarrhoea in IBS. However, abdominal pain, bloating, nausea and constipation are common, and may limit tolerability. Titrating the dose carefully may avoid this (recommendation: strong; quality of evidence: very low).

- Certain antispasmodics may be an effective treatment for global symptoms and abdominal pain in IBS. Dry mouth, visual disturbance and dizziness are common side effects (recommendation: weak, quality of evidence: very low).

- Peppermint oil may be an effective treatment for global symptoms and abdominal pain in IBS. Gastro-oesophageal reflux is a common side effect (recommendation: weak, quality of evidence: very low).

- Polyethylene glycol may be an effective treatment for constipation in IBS. Abdominal pain is a common side effect (recommendation: weak; quality of evidence: very low).

\section{Gut-brain neuromodulators}

Dysfunction within the bidirectional gut-brain axis is considered to play an important role in the genesis and maintenance of symptoms in IBS. Although IBS is often considered a functional gastrointestinal disorder, these conditions have, therefore, been re-termed as disorders of gut-brain interaction. ${ }^{32}$ Patients with IBS often have comorbid anxiety and depression, ${ }^{119}$ and these are also risk factors for the subsequent development of IBS in healthy people. ${ }^{31}$ This, together with their peripheral effects on gastrointestinal function, ${ }^{223}$ is part of the rationale for the use of gut-brain neuromodulators, such as TCAs and SSRIs. In a metaanalysis of 12 RCTs of TCAs, recruiting 787 patients, these drugs were superior to placebo for global symptoms or abdominal pain (RR $0.65 ; 95 \%$ CI 0.55 to 0.77 ) (online supplemental figure 8), and for abdominal pain alone (RR 0.59 ; 95\% CI 0.42 to 0.83 ) (online supplemental figure 9). ${ }^{159}$ SSRIs were also more efficacious for global symptoms or abdominal pain (RR 0.68 ; 95\% CI
0.51 to 0.91 ) (online supplemental figure 8 ), but not abdominal pain alone (online supplemental figure 9), and there was significant heterogeneity among the 7 trials, which contained only 356 patients. ${ }^{159}$ Adverse event rates were significantly higher among patients treated with TCAs or SSRIs, with drowsiness and dry mouth the most common. ${ }^{159}$ The effect of these drugs on stool pattern is less clear, as very few trials restricted their recruitment to a particular subgroup of patients.

Other gut-brain neuromodulators include serotonin norepinephrine reuptake inhibitors (SNRIs), such as duloxetine, or agents acting on the calcium channel $\alpha 2 \delta$ ligand, including pregabalin. There have been no RCTs of SNRIs in IBS, although there is evidence from case series that duloxetine may improve symptoms and quality of life, ${ }^{224225}$ and there is good evidence for use of SNRIs in other chronic painful disorders, such as fibromyalgia and low back pain. ${ }^{226}$ Pregabalin improved visceral hypersensitivity in one small trial, ${ }^{227}$ and in a recent RCT recruiting 85 patients with IBS, 12 weeks of pregabalin 225 mg twice daily led to significant improvements in global symptoms, abdominal pain, diarrhoea and bloating versus placebo. ${ }^{228}$ Blurred vision, dizziness and altered sensation were more common with pregabalin. However, more RCTs are needed, and pregabalin is classed as a controlled drug in some countries. In a network meta-analysis evaluating relative efficacy of gutbrain modulators with other unlicensed or 'traditional' treatments, ${ }^{157}$ TCAs were ranked second and first for their effect on global symptoms and abdominal pain respectively, and were more efficacious than placebo (online supplemental figures 6 and 7). In contrast, SSRIs were ranked fifth and fourth for global symptoms and abdominal pain, respectively, and pregabalin was ranked sixth for global symptoms, with no benefit of either over placebo in these trials.

It is reasonable to consider using TCAs second line to treat global symptoms or abdominal pain or SSRIs second line to treat global symptoms, or if there is coexistent anxiety. ${ }^{226}$ They can be offered by general practitioners, depending on familiarity of use and expertise. The rationale for the use of gut-brain neuromodulators, as well as their side-effect profile, needs to be explained carefully to the patient, within the context of IBS as a disorder of gut-brain interaction. ${ }^{226}$ It should be reinforced that these drugs are being used at low doses for their pain modulatory properties and peripheral effects on gastrointestinal function, rather than at a dose that is used to treat common mental disorders. TCAs should be taken in the evening, before bedtime, due to their sedating effects, and may also improve sleep patterns. The patient should be counselled that these drugs take some time to have any benefit and that side effects, such as drowsiness, tend to ameliorate after the first 1 or 2 weeks of treatment. They should be commenced at a low dose (eg, $10 \mathrm{mg}$ of amitriptyline once a day) and titrated relatively slowly in $10 \mathrm{mg}$ increments, to a maximum of 30-50 mg once a day, with follow-up to assess efficacy and tolerability. If beneficial, the drugs are likely to be continued for a minimum of 6-12 months and, in some cases, this may be even longer-term.

\section{Recommendations}

- Tricyclic antidepressants used as gut-brain neuromodulators are an effective second-line drug for global symptoms and abdominal pain in IBS. They can be initiated in primary or secondary care, but careful explanation as to the rationale for their use is required, and patients should be counselled about their side-effect profile. They should be commenced at a low dose (eg, $10 \mathrm{mg}$ amitriptyline once a day) and titrated 
slowly to a maximum of 30-50 mg once a day (recommendation: strong, quality of evidence: moderate).

- Selective serotonin reuptake inhibitors used as gut-brain neuromodulators may be an effective second-line drug for global symptoms in IBS. As with tricyclic antidepressants, they can be initiated in primary or secondary care, but careful explanation as to the rationale for their use is required, and patients should be counselled about their side-effect profile (recommendation: weak, quality of evidence: low).

\section{Drugs used second line for the treatment of IBS-D}

For patients with IBS-D who do not experience symptom improvement with antidiarrhoeals, several licensed therapies are available in secondary care. Eluxadoline is a $\mu$-opioid and $\kappa$-opioid receptor agonist and $\delta$-opioid receptor antagonist licensed for IBS-D. The drug slows intestinal transit and reduces visceral hypersensitivity. ${ }^{229}$ Data from a meta-analysis (4 RCTs containing 3122 patients) demonstrated that both $75 \mathrm{mg}$ two times per day and $100 \mathrm{mg}$ two times per day were superior to placebo using the FDA-approved composite end point for IBS-D, consisting of improvement in abdominal pain and stool consistency (RR 0.89 ; 95\% CI 0.84 to 0.94 and RR 0.87 ; 95\% CI 0.83 to 0.91 , respectively) (online supplemental figure 10 ), global symptoms and stool consistency (online supplemental figures 11 and 12). ${ }^{153}$ Eluxadoline $100 \mathrm{mg}$ two times per day was also superior to placebo for abdominal pain (online supplemental figure 13). Adverse events included constipation, nausea and headache, and adverse events leading to drop out were significantly higher with active drug than placebo. Serious adverse events, including pancreatitis and sphincter of Oddi spasm, have been reported, occurring in $0.5 \%$ of patients in these trials. ${ }^{230}$ The drug is contraindicated in patients with prior sphincter of Oddi problems or cholecystectomy, alcohol dependence, pancreatitis or severe liver impairment. Although licensed for IBS-D, the drug is unavailable in many countries.

Drugs acting as antagonists at the $5-\mathrm{HT}_{3}$ receptor are also licensed for IBS-D. These include alosetron and ramosetron, which slow gastrointestinal transit, reduce visceral hypersensitivity and alter rectal compliance. ${ }^{231-233}$ In a previous metaanalysis, ${ }^{153}$ both alosetron $1 \mathrm{mg}$ two times per day and ramosetron $2.5 \mu \mathrm{g}$ or $5 \mu \mathrm{g}$ once a day were superior to placebo across various end points, including the FDA composite end point for IBS-D (3 RCTs of alosetron $1 \mathrm{mg}$ two times per day, 787 patients, RR 0.69 ; $95 \%$ CI 0.60 to 0.80 , and 1 RCT of ramosetron $2.5 \mu \mathrm{g}$ once a day, 348 patients, RR 0.78 ; 95\% CI 0.67 to 0.91 ) (online supplemental figure 10). Both drugs were also more efficacious than placebo for global symptoms, abdominal pain and stool consistency (online supplemental figures 11-13). Adverse events included constipation, nausea and headache; patients assigned to both drugs were more likely to report adverse events than with placebo. Alosetron was withdrawn from the market in 2001 due to reports of ischaemic colitis. ${ }^{234}$ However, it was reintroduced in the USA via a risk evaluation and mitigation strategy, at a lower dose of $0.5 \mathrm{mg}$ two times per day, for women with severe IBS-D. Rates of ischaemic colitis observed since reintroduction are similar to the background rate in female patients with IBS. ${ }^{235}$ Ramosetron is only available in Asia. ${ }^{153}$ There have been no reports of ischaemic colitis associated with the drug. Due to the limited availability of both alosetron and ramosetron, RCTs of ondansetron, a widely available $5-\mathrm{HT}_{3}$ receptor antagonist with a robust safety profile, have been conducted. A small crossover trial of ondansetron titrated from $4 \mathrm{mg}$ once a day to a maximum of $8 \mathrm{mg}$ three times a day demonstrated significantly higher rates of improvement in urgency, bloating and stool consistency, but not abdominal pain. ${ }^{236} \mathrm{~A}$ subsequent RCT of $12 \mathrm{mg}$ once a day of bimodal release ondansetron also demonstrated superiority over placebo for improvement in stool consistency, but not abdominal pain. ${ }^{178}$ Constipation is the most common side effect. Results from a parallel-group RCT are awaited. ${ }^{237}$

The efficacy of rifaximin, a non-absorbable antibiotic, has also been tested in IBS-D, on the basis that disturbances in the gastrointestinal microbiota may, in part, be responsible for symptoms. In a meta-analysis of 2 RCTs, ${ }^{153}$ which recruited 1260 patients, rifaximin $550 \mathrm{mg}$ three times a day for 14 days was more efficacious than placebo for the FDA composite end point for IBS-D (RR $0.92 ; 95 \%$ CI 0.86 to 0.98 ) (online supplemental figure 10 ) and for stool consistency alone (online supplemental figure 12), but not for global symptoms or abdominal pain (online supplemental figures 11 and 13). Headache was the most common adverse event, but side effects were no more common with rifaximin than with placebo. Due to the modest efficacy, and concerns over the potential for adverse events (including Clostridium difficile infection and bacterial resistance) with repeated courses of rifaximin, FDA approval was not forthcoming. A subsequent 'retreatment' trial was therefore conducted. In this RCT, patients received open-label rifaximin and were then randomised to two repeat 14-day courses of rifaximin or placebo if they experienced symptom relapse. Significantly more patients experienced an improvement in global symptoms with rifaximin after each treatment course, and there were no safety concerns. ${ }^{238}$ The drug is now licensed for IBS-D in the USA but is not available for this indication in many countries.

A network meta-analysis comparing the relative efficacy of all these licensed therapies for IBS-D, across various end points, ${ }^{153}$ demonstrated that alosetron $1 \mathrm{mg}$ two times per day ranked first for the FDA composite end point for IBS-D and global symptoms (online supplemental figures 10 and 11), with ramosetron $2.5 \mu \mathrm{g}$ once a day second. For the FDA composite end point, alosetron was superior to all treatments, except ramosetron 2.5 $\mu \mathrm{g}$ once a day For, abdominal pain, ramosetron $2.5 \mu \mathrm{g}$ once a day and ramosetron $5 \mu \mathrm{g}$ once a day were ranked first and second, respectively (online supplemental figure 13). Finally, for stool consistency alosetron $1 \mathrm{mg}$ two times per day ranked first, with ramosetron $5 \mu \mathrm{g}$ once a day second (online supplemental figure 12).

\section{Recommendations}

- Eluxadoline, a mixed opioid receptor drug, is an efficacious second-line drug for IBS with diarrhoea in secondary care. It is contraindicated in patients with prior sphincter of Oddi problems or cholecystectomy, alcohol dependence, pancreatitis or severe liver impairment, and lack of availability may limit its use (recommendation: weak, quality of evidence: moderate).

- 5-Hydroxytryptamine 3 receptor antagonists are efficacious second-line drugs for IBS with diarrhoea in secondary care. Alosetron and ramosetron are unavailable in many countries; ondansetron titrated from a dose of $4 \mathrm{mg}$ once a day to a maximum of $8 \mathrm{mg}$ three times a day is a reasonable alternative. Constipation is the most common side effect. This drug class is likely the most efficacious for IBS with diarrhoea (recommendation: weak, quality of evidence: moderate to high).

- The non-absorbable antibiotic rifaximin is an efficacious second-line drug for IBS with diarrhoea in secondary care, although its effect on abdominal pain is limited. The drug is 
licensed for IBS with diarrhoea in the USA but is not available for this indication in many countries (recommendation: weak, quality of evidence: moderate).

\section{Drugs used second line for the treatment of IBS-C}

In patients with IBS-C who do not experience symptom improvement with laxatives, escalation to second-line drugs should be considered in secondary care. These fall into two main classes, secretagogues and 5- $\mathrm{HT}_{4}$ agonists. Secretagogues, including linaclotide, lubiprostone, plecanatide and tenapanor activate ion channels on the intraluminal surface of enterocytes, resulting in an efflux of ions and water into the intestinal lumen, softening stools and accelerating transit. ${ }^{239} 240$ Linaclotide is a peptide that acts as a guanylate cyclase- $\mathrm{C}$ agonist. Lubiprostone is a prostaglandin $\mathrm{E}_{1}$ derivative, which activate chloride type 2 channels. Plecanatide is another guanylate cyclase- $\mathrm{C}$ agonist that binds in a pH-dependent manner, in contrast to linaclotide, such that the majority of its activity is confined to the proximal small bowel. ${ }^{241}$ Tenapanor is a small molecule inhibitor of the gastrointestinal sodium-hydrogen exchanger-3. 5- $\mathrm{HT}_{4}$ agonists, such as tegaserod, have prokinetic effects and also accelerate transit. ${ }^{242}$ Abdominal bloating is a particularly troublesome symptom in patients with IBS-C, ${ }^{46}$ and the effects of all these drugs on this symptom, other than plecanatide, has been assessed in some RCTs.

In an update of a previous meta-analysis, ${ }^{155}$ a dose of 290 $\mu \mathrm{g}$ once a day linaclotide was superior to placebo in 5 RCTs, containing 3193 patients, for the FDA composite end point for IBS-C, consisting of improvement in abdominal pain and an increase of $\geq 1$ CSBMs per week from baseline (RR 0.82; 95\% CI 0.78 to 0.87 ) (online supplemental figure 14), abdominal pain alone (online supplemental figure 15) and an increase of $\geq 1$ CSBMs per week from baseline (online supplemental figure 16). The drug was also superior to placebo in terms of an improvement in abdominal bloating in 4 trials containing 3061 patients (online supplemental figure 17). Adverse events were significantly more common with linaclotide $290 \mu \mathrm{g}$ once a day, with diarrhoea being the most common. Lubiprostone $8 \mu \mathrm{g}$ two times per day was superior to placebo for both the FDA composite end point for IBS-C (RR 0.87; 95\% CI 0.78 to 0.96) (online supplemental figure 14) and abdominal pain alone (online supplemental figure 15) in this meta-analysis, using a post hoc analysis of data from 2 phase III RCTs, containing 452 patients. ${ }^{155}$ The drug was superior to placebo for abdominal bloating in these 2 RCTs (online supplemental figure 17). Adverse events were no more common with lubiprostone, except for nausea. In the same meta-analysis, ${ }^{155}$ both plecanatide $3 \mu \mathrm{g}$ once a day and 6 $\mu \mathrm{g}$ once a day were superior to placebo for the FDA composite end point (RR $0.88 ; 95 \%$ CI 0.82 to 0.94 for $3 \mu \mathrm{g}$ once a day in 3 RCTs, recruiting 1632 patients, and RR 0.87; 95\% CI 0.81 to 0.93 for $6 \mu \mathrm{g}$ once a day in 2 RCTs, containing 1461 patients) (online supplemental figure 14) and abdominal pain alone (online supplemental figure 15), but not for an increase of $\geq 1$ CSBMs per week from baseline (online supplemental figure 16). Adverse events were significantly more frequent with plecanatide $3 \mu \mathrm{g}$ once a day, compared with placebo, and diarrhoea was significantly more likely with both doses. Finally, 3 RCTs of tenapanor $50 \mathrm{mg}$ two times per day, recruiting 1428 patients, were included in this meta-analysis. ${ }^{155}$ The RR for the FDA composite end point, compared with placebo, was 0.85 (95\% CI 0.79 to 0.92 ) (online supplemental figure 14). The drug was also more efficacious than placebo for abdominal pain (online supplemental figure 15 ) and an increase of $\geq 1$ CSBMs per week from baseline (online supplemental figure 16). Again, the drug was more likely to improve abdominal bloating than placebo in 3 trials containing 1428 patients (online supplemental figure 17). Except for diarrhoea, adverse events were no more likely with the drug than with placebo.

A previous meta-analysis of 11 RCTs demonstrated that tegaserod was superior to placebo for the treatment of IBS-C in 9242 patients (RR 0.85 ; 95\% CI 0.80 to 0.90 ) (online supplemental figure 18). ${ }^{163}$ Diarrhoea was the most common adverse event and was significantly more likely than with placebo. Due to a small excess number of cerebrovascular and cardiovascular ischaemic events in patients taking the drug, it was withdrawn in 2007. Tegaserod was reintroduced in the USA in 2018 for female patients $<65$ years of age with IBS-C without pre-existing cardiovascular disease, based on a post hoc analysis of 3 large trials reporting efficacy according to the FDA composite end point for IBS-C. In a meta-analysis using data from these 3 trials, containing 2472 patients, the drug was superior to placebo (RR $0.85 ; 95 \%$ CI 0.80 to 0.91 ) (online supplemental figure 14 ). ${ }^{154}$ Finally, tegaserod was superior to placebo for abdominal bloating in 4 RCTs, containing 5132 patients (online supplemental figure 17). Although prucalopride, which is a highly selective $5-\mathrm{HT}_{4}$ agonist with no known cardiovascular or cerebrovascular safety concerns, is efficacious in the treatment of chronic idiopathic constipation, ${ }^{220}$ to date there have been no RCTs in IBS-C.

A network meta-analysis examining the relative efficacy of secretagogues and tegaserod across 18 RCTs, in 10638 patients, demonstrated that all drugs were superior to placebo. ${ }^{154} 155$ Linaclotide $290 \mu \mathrm{g}$ once a day ranked first across all end points, including abdominal bloating (online supplemental figure 14-16 and online supplemental figure 19), but on indirect comparison of active treatments there were no significant differences between individual drugs and dosages.

\section{Recommendations}

- Linaclotide, a guanylate cyclase-C agonist, is an efficacious second-line drug for IBS with constipation in secondary care. It is likely to be the most efficacious secretagogue available for IBS with constipation, although diarrhoea is a common side effect (recommendation: strong, quality of evidence: high).

- Lubiprostone, a chloride channel activator, is an efficacious second-line drug for IBS with constipation in secondary care. This secretagogue is less likely to cause diarrhoea than others. However, patients should be warned that nausea is a frequent side effect (recommendation: strong, quality of evidence: moderate).

- Plecanatide, another guanylate cyclase-C agonist, is an efficacious second-line drug for IBS with constipation in secondary care. Diarrhoea is a common side effect and is no less likely than with linaclotide or tenapanor. Although the drug is licensed for IBS with constipation in the USA, it is not yet available for this indication in many countries (recommendation: strong, quality of evidence: high).

- Tenapanor, a sodium-hydrogen exchange inhibitor, is an efficacious second-line drug for IBS with constipation in secondary care. Again, diarrhoea is a frequent side effect. Although the drug is licensed for IBS with constipation in the USA, it is not yet available for this indication in many countries (recommendation: strong, quality of evidence: high).

- Tegaserod, a 5-Hydroxytryptamine 4 receptor agonist, is an efficacious second-line drug for IBS with constipation in 
secondary care but is unavailable outside the USA. Diarrhoea is a common side effect (recommendation: strong, quality of evidence: moderate).

\section{Psychological therapies}

A recent network meta-analysis of RCTs of psychological therapies for IBS demonstrated that several psychological therapies were more efficacious than control interventions. ${ }^{156}$ However, the most compelling evidence, based on the number of trials and long-term outcomes was for CBT and gut-directed hypnotherapy, ${ }^{156}$ both of which are recommended by the NICE guideline when symptoms have not improved after 12 months of drug treatment. $^{10}$

The principles of CBT are based on the five systems model, which suggests that cognitions (thoughts), behaviours (actions), emotions and physiology all interact within the context of the broader environment or social system. By altering any of these systems, others can potentially be modified (eg, changing one's thoughts can alter one's emotions, as well as one's physiological responses). Although there is a core set of defined therapeutic techniques employed in all variants of CBT, the underlying formulations of the therapy differ, depending on the primary outcome. For instance, if reducing depression is the primary outcome the therapy focuses on increasing pleasurable activities and challenging alternative negative thoughts about the self. If anxiety is the outcome, the therapy focuses on reducing avoidance of threatening situations and threat-related thought patterns.

The network meta-analysis of psychological interventions for IBS, which included 15 trials of CBT in 1844 patients, concluded that CBT delivered in several formats was more effective than a control, including education and support, treatment as usual, and a waiting list control (online supplemental figure 20). ${ }^{156}$ Face-face CBT (10 RCTs, 930 patients, RR 0.62; 95\% CI 0.48 to 0.80 ), self-administered or minimal contact CBT (4 trials, 434 patients, RR 0.61 ; 95\% CI 0.45 to 0.83 ), therapistdelivered CBT over the telephone (1 RCT, 373 patients, RR $0.50 ; 95 \%$ CI 0.29 to 0.84 ) and group CBT ( 2 trials, 50 patients, RR 0.41 ; $95 \%$ CI 0.19 to 0.91 ) were all superior to a waiting list control. ${ }^{156}$ There was substantial heterogeneity in some of the estimates, which may, in part, be explained by differences in trial design, sample size and whether patients with refractory IBS were included. Analysis of trials that only included patients with refractory symptoms reduced the heterogeneity, and still demonstrated efficacy for CBT in some formats (online supplemental figure 21).

Other sources of heterogeneity may include hours of therapy time. For example, within the face-to-face CBT groups therapist time ranged from 5 to 12 hours. Of the two web-based interventions, one had eight online interactive sessions with 2.5 hours of telephone therapist support, and the other five online sessions with email support. There were also differences in the skill level of therapists across trials. Most were experienced CBT therapists, but some trials used doctoral-level students, and one RCT trained nurses to deliver CBT. The CBT protocols themselves varied. Some made IBS-specific modifications to existing mental health protocols, and others were based on stress management related to IBS.

The 2 most recent, and largest, RCTs used CBT developed specifically for IBS. ${ }^{86} 243$ In both, this included education concerning the role of stress in IBS, stress management techniques, cognitive techniques to identify and challenge both unhelpful thoughts associated with IBS and core beliefs around perfectionism, and relapse prevention. The ACTIB trial also included a description of the pathophysiology of key symptoms, and how CBT may work through the gut-brain axis. ${ }^{86}$ Other sections focused on altering IBS-specific safety and avoidance behaviours (eg, not going out until bowels are empty, or a toilet location is known) and managing negative emotions. The IBSOS trial included problem-solving training focused on coping with IBS stressors. ${ }^{243}$

The rationale for IBS-specific CBT is further supported by a review of the psychological mechanisms of CBT for IBS. ${ }^{30}$ Key mechanisms related to reduction in IBS symptom severity appear to be changes in IBS-specific cognitions and gastrointestinal -specific anxiety, rather than changes in general anxiety. With respect to this issue, it is worth noting that in the largest trial of CBT for IBS conducted to date at least $50 \%$ of patients met cutoffs for probable common mental disorders at baseline, and both therapist-delivered CBT over the telephone and web-based CBT using IBS-specific protocols reduced anxiety and depression scores at all follow-up points. ${ }^{86}$ These data suggest that treatment with IBS-specific CBT protocols may benefit both mental health and gastrointestinal symptoms.

The network meta-analysis suggested that therapist-delivered CBT over the telephone had a larger effect on IBS symptoms at follow-up than web-based CBT. ${ }^{156}$ However, in the ACTIB trial, health economic analysis suggested web-based CBT was the more cost-effective option. ${ }^{244}$ In this trial, the web-based intervention used the same protocol as the therapist-delivered CBT over the telephone, ${ }^{244}$ but the eight sessions were delivered on an interactive, tailored, website. Patients worked through this on their own at home, with guided telephone support from the therapist. The network meta-analysis also demonstrated that therapist-delivered CBT over the telephone, web-based CBT, face-to-face CBT and self-administered or minimal contact CBT were all superior to treatment as usual after 12 months of follow-up (online supplemental figure 22). ${ }^{156}$ It is likely that these approaches have similar efficacy, but more work is needed to determine cost-effectiveness of the various modes of delivery. One advantage of web-based therapies is that they are easy to standardise at scale and monitor usage. However, the disadvantage is that they tend to have lower adherence. ${ }^{86244}$

Evidence suggests, therefore, that CBT for IBS is effective in both high-intensity (therapist-delivered) and minimal contact (therapist-guided) formats, as well as self-administered with either bibliographic material or web-based. There is also some evidence for group CBT, but more trials are needed to confirm this. As the ACTIB trial suggested a bigger treatment effect with therapist-delivered treatment, but that this was less likely to be cost-effective, ${ }^{244}$ a stepped care approach may provide greatest benefit, where patients with more complex needs receive faceto-face CBT, and those with milder symptoms are offered webbased or other guided, supported versions.

Despite an evidence base for use, ${ }^{156}$ many psychological therapies are not widely available, despite being recommended in the NICE guideline for patients with ongoing symptoms after 12 months of drug treatment. ${ }^{10}$ However, with the success of the ACTIB and IBSOS trials, ${ }^{86} 243244$ there have been improved training opportunities for therapists, and therapist manuals made freely available to Improving Access to Psychological Therapy (IAPT) services in the UK, on completion of a specified training programme. Telephone and web-based delivery of CBT also has the potential to further increase access. ${ }^{86}$ The IAPT service has, therefore, increased its remit to include CBT for IBS, and patients can be referred via general practitioners or can self-refer. More work is needed to enhance and standardise 
the training programme providers to ensure IAPT expertise in this area. Therapists without specific IBS training tend to default to using mental health treatments, which can disengage patients with IBS. Therefore, referrals should specify that this is for IBSspecific CBT.

Gut-directed hypnotherapy is one of the psychological therapies for IBS with the largest evidence base for both short-term and long-term efficacy in RCTs. ${ }^{156}$ The aims of this are to induce a deep state of relaxation in order to teach the patient new skills for self-management and control of their gut function. ${ }^{245}$ The treatment is delivered using IBS-specific protocols, ${ }^{245} 246$ which incorporate combinations of a variety of techniques including imagery, metaphors, tactile approaches to alleviate pain and diaphragmatic breathing specifically targeting abdominal bloating and distension. One of the strengths of the treatment is that the content can be tailored according to the patient's symptom profile. Although the exact mechanisms of its effects in IBS remain uncertain, hypnotherapy modulates the gutbrain axis, with several studies demonstrating positive changes in gut-brain function before, and immediately after, hypnotherapy, including modulation of postprandial gastro-colic reflex activity, ${ }^{247}$ altered colonic motility, ${ }^{248}$ reduced visceral hypersensitivity $^{249}$ and normalisation of gut-brain pain processing signals on functional brain imaging. ${ }^{250} 251$

Traditionally, hypnotherapy for IBS has been delivered via between 6 and 12 face-to-face weekly sessions of individualised treatment with a trained therapist. This approach has been shown to be efficacious; a meta-analysis of $6 \mathrm{RCTs}$, recruiting 639 patients, reported a RR of remaining symptomatic of 0.73 (95\% CI 0.55 to 0.97 ) compared with education and/or support and 0.67 (95\% CI 0.49 to 0.91 ) compared with a waiting list control (online supplemental figure 20). ${ }^{156}$ Moreover, in the largest clinical series to date, including 1000 patients, $>75 \%$ of patients achieved a clinical response to hypnotherapy, defined as a $\geq 50$-point reduction in IBS symptom severity score. There were also significant improvements in extraintestinal symptoms, and anxiety and depression scores. ${ }^{252}$ Hypnotherapy has previously only been recommended for patients with IBS when symptoms are refractory to conventional treatments. ${ }^{10}$ Indeed, a meta-analysis of RCTs has shown that gut-directed hypnotherapy is one of the few treatments that performs better than a control for patients with refractory symptoms (online supplemental figure 21). ${ }^{156}$ However, its clinical efficacy has also been demonstrated in non-refractory populations, ${ }^{156}$ and clinical outcomes in children and adolescents with IBS suggest that use of gut-directed hypnotherapy at an earlier stage of the condition may be beneficial. ${ }^{253}$

One of the barriers to wider scale provision of gut-directed hypnotherapy, and its current restriction to refractory cases, may be the cost of its delivery, including time intensity, and the requirement for a trained therapist. However, intervention with gut-focused hypnotherapy has been shown to have wider socioeconomic benefits including improving general well-being, reductions in healthcare utilisation in both primary and secondary care, ${ }^{254-256}$ reduced presenteeism at work, ${ }^{255}$ improved quality of life $e^{252} 257$ and long-term beneficial effects on symptoms, ${ }^{256}$ making it a potentially cost-effective option. Patients with IBS in tertiary care with severe functional limitations may require individualised hypnotherapy, with the content of sessions customised to their symptom profiles. However, patients in primary or secondary care may benefit from accessing a more 'generic' form of group-delivered hypnotherapy. In a large, multicentre, RCT in patients with IBS in primary or secondary care, group hypnotherapy was shown to be non-inferior to individual hypnotherapy. ${ }^{258}$ Group hypnotherapy may therefore have a role in primary and secondary care settings, with the potential advantage that this approach could reduce delivery costs and improve access. Early reports of clinical outcomes via videoconsultation are also promising, with similar response rates achieved, compared with face-to-face treatment. ${ }^{259}$

The offer of psychological therapies should not be limited to patients with psychological comorbidities. IBS symptoms are inherently distressing so there are often symptoms of anxiety and depression in IBS, but these are not necessarily at case level for a psychiatric diagnosis. Nevertheless, it may be worthwhile screening for evidence of both in the clinic, using a simple questionnaire, such as the General Anxiety Disorder assessment-7 and the Patient Health Questionnaire-9, and discussing referral to a mental health service or psychiatrist if mood is felt to be the key issue. The majority of the trials of psychological therapies conducted to date include a wide range of patients with IBS, many of whom would also not have met the threshold for a psychiatric disorder. In contrast to psychological treatments used for psychiatric disorders, which focus on mood, the IBSspecific therapies discussed here focus on brain-gut symptomspecific treatment mechanisms. The primary aim of treatment is to reduce severity and impact of abdominal pain and to help regulate bowel habit. They should, therefore, be viewed as behavioural methods for managing and treating IBS symptoms, rather than as psychotherapies.

\section{Recommendations}

- IBS-specific cognitive behavioural therapy may be an efficacious treatment for global symptoms in IBS (recommendation: strong, quality of evidence: low).

- Gut-directed hypnotherapy may be an efficacious treatment for global symptoms in IBS (recommendation: strong, quality of evidence: low).

- Psychological therapies should be considered when symptoms have not improved after 12 months of drug treatment. Referral can be made at an earlier stage, if accessible locally, and based on patient preference (recommendation: strong, quality of evidence: low).

\section{Approach to the patient with severe or refractory symptoms}

Severe IBS lacks a precise consensus definition, but is considered to be a biopsychosocial composite of patient-reported gastrointestinal and extraintestinal symptoms, degree of disability, illness-related perceptions and behaviours, ${ }^{260}$ insufficient response to conventional treatments ${ }^{261}$ and high healthcare utilisation. ${ }^{262}$ Refractory IBS is a related, but distinct term, again with no consensus definition, which is taken to mean patients whose symptoms have not improved with interventions, some of whom may also have severe symptoms. Validated severity scoring systems to assess impact and severity of IBS symptoms include the IBS severity scoring system, ${ }^{263}$ the gastrointestinal symptom rating scale-IBS ${ }^{264}$ and the functional bowel disorder severity index. ${ }^{265}$ In one European study, approximately one in four individuals with IBS were categorised as severe in a general population setting. ${ }^{266}$

Although the risk of missing, or subsequently developing, an organic disorder in patients diagnosed with IBS is low, this rate may be increased in those with severe symptoms, ${ }^{267}$ and should prompt a review of the diagnosis, with consideration of further targeted investigation. Nevertheless, it is important to stress that in most patients a diagnosis of IBS is secure, ${ }^{111}$ and further repeated investigations have a low yield. ${ }^{268}$ Severe IBS 
should also be distinguished from other severe functional gastrointestinal disorders that may have overlapping symptom presentations, including the narcotic bowel syndrome, if the patient is taking long-term opioids, centrally mediated abdominal pain syndrome ${ }^{269}$ and small intestinal dysmotility. ${ }^{270}$ Referral to a multidisciplinary chronic pain team to aid pain management and help with opioid reduction should be considered if abdominal pain becomes centrally-mediated or if narcotic bowel syndrome develops.

A large and diverse range of dietary, microbial, traumatic, interpersonal, genetic, psychological, physiological, psychiatric and functional comorbidity factors have been described in patients with severe IBS. ${ }^{75} 189271-276$ Consequently, it is unlikely that a single targeted intervention will be transformative and a multidimensional, multisystem and integrated multidisciplinary team approach is usually required. Although there is a limited evidence base to guide management of this group of patients, as most RCTs do not differentiate response to treatment according to baseline symptom severity, there is recent trial data reporting superior outcomes with an integrated approach involving gastroenterologists, dietitians and clinical psychologists, rather than a gastroenterologist alone. ${ }^{277}$ There is a danger that, in this vacuum, 'alternative' therapies with the least evidence for their efficacy and safety are recommended by physicians or other practitioners, or are sought out by patients. ${ }^{278}$

Patients with IBS with severe symptoms are more willing to accept significant medication risks, for example, a mean 1\% chance of sudden death in return for a $99 \%$ chance of cure of their symptoms with a hypothetical medication. ${ }^{279}$ Furthermore, patients are at increased risk of iatrogenic harms, through unnecessary surgery, including hysterectomy, appendicectomy and cholecystectomy, ${ }^{121}$ and inappropriate prescribing of opioids. ${ }^{90}$ The first principle of care for this vulnerable population of patients should therefore be primum non-nocere-first do no harm. This includes harm from unregulated and unproven approaches, especially if incentivised by financial or reputational gain.

Interventions with reported efficacy for patients with undifferentiated IBS specifically classified as severe or refractory include CBT, integrative group therapy, gut-directed hypnotherapy, gut-brain neuromodulators or psychodynamic interpersonal therapy. ${ }^{86} 243252$ 280-282 For severe or refractory IBS-C, surgical management, consisting of a potentially reversible temporary loop ileostomy, with a view to colectomy if stoma function results in improved, rather than worsened, quality of life for the appropriately screened patient, ${ }^{139}$ the ileal bile acid transporter inhibitor elobixibat, ${ }^{283}$ or linaclotide, ${ }^{284}$ all have some evidential support. For severe or refractory IBS-D, alosetron has regulatory approval in the USA for women. ${ }^{285}$ In the UK, a reasonable alternative might be ondansetron although, to date, this has not yet been confirmed to be efficacious in severe or refractory IBS. For severe or refractory abdominal pain one study reported efficacy with intramuscular hyoscine. ${ }^{286}$

Other IBS guidelines suggest the use of combination gut-brain neuromodulators, termed augmentation, for more severe symptoms. ${ }^{226}$ Evidence from a large cohort of patients with severe chronic continuous abdominal pain showed that combinations of neuropathic analgesics (eg, duloxetine plus gabapentin) were more efficacious than monotherapy. ${ }^{287}$ Vigilance for the development of the serotonin syndrome for some combinations, especially those involving both SSRIs and SNRIs, is required. Symptoms include fever, hyperreflexia, tremor, sweating and diarrhoea. For patients with symptoms that are refractory to these pharmacological therapies, and those who have comorbid conditions or psychological symptoms, a combination of a gutbrain neuromodulator and psychological therapy may be more efficacious than monotherapy with either, drawing parallels with evidence from the depression and chronic headache literature. ${ }^{288} 289$

\section{Recommendations}

- Severe or refractory IBS symptoms should prompt a review of the diagnosis, with consideration of further targeted investigation (recommendation: weak, evidence: very low).

- Severe or refractory IBS should be managed with an integrated multidisciplinary approach (recommendation: weak, evidence: very low).

- Iatrogenic harms due to opioid prescribing, unnecessary surgery and unproven unregulated diagnostic or therapeutic approaches incentivised by financial or reputational gain should be avoided (recommendation: strong, evidence: very low).

- Use of combination gut-brain neuromodulators, termed augmentation, may be considered for more severe symptoms, with vigilance for risks of serotonin syndrome (recommendation: weak, evidence: very low).

\section{Drugs in development}

The highly selective $5-\mathrm{HT}_{4}$ agonist minesapride has been studied in 2 phase II dose-ranging RCTs in patients with IBS-C. ${ }^{179} 180$ A dose of $40 \mathrm{mg}$ once a day was superior to placebo, in terms of improvements in number of bowel movements per week, abdominal pain and global symptoms. The drug was welltolerated, with diarrhoea the most common side effect, and there were no cardiovascular adverse events. Histamine has a potential role in mediating visceral hypersensitivity, and in a small RCT in 45 patients the histamine ${ }_{1}$ receptor antagonist ebastine led to significant improvements in both abdominal pain and global symptoms. ${ }^{290} \mathrm{~A}$ larger trial in 200 patients is ongoing (NCT01908465). Novel drugs that have been tested successfully in chronic idiopathic constipation, including elobixibat and mizagliflozin, a sodium-glucose cotransporter-1 inhibitor, are likely to undergo testing in IBS-C. ${ }^{283}{ }^{291}$ Some secretagogues, including linaclotide, stimulate cyclic GMP production, which can attenuate visceral pain. ${ }^{292}$ An RCT of delayed-release linaclotide, with action confined to the ileo-caecal region of the gastrointestinal tract, demonstrated significant effects on abdominal pain, with lower rates of diarrhoea than conventionrelease linaclotide. ${ }^{165}$ Other novel approaches include drugs that act on cannabinoid receptors, which are expressed in the gastrointestinal tract and may also modulate pain expression. The cannabinoid type- 2 receptor agonist, olorinab, has been tested in patients with quiescent Crohn's disease, and led to reductions in abdominal pain and improved bowel movements ${ }^{293}$; a trial in IBS is underway (NCT04043455).

\section{Other treatments in development}

In recent years, there has been considerable interest in the evaluation of faecal microbiota transplantation (FMT) for IBS. Unfortunately, a meta-analysis of 5 RCTs, containing 267 patients, demonstrated no significant benefit of FMT compared with placebo (RR $0.98 ; 95 \%$ CI 0.58 to 1.66 ), and in 2 pooled trials placebo capsules administered orally were superior to capsules containing donor stool (RR 1.96; $95 \%$ CI 1.19 to 3.20 ). ${ }^{294}$ Criticisms of the trials, to date, have included small sample sizes, heterogeneity in IBS subtypes recruited, lack of standardisation of donor samples and suboptimal end points used. There is 
therefore a need for further, large, high-quality trials of FMT for IBS, perhaps targeting subgroups of patients with evidence of dysbiosis, who may be more likely to benefit. At present, therefore, there is insufficient evidence to recommend FMT for IBS outside of a research setting. Enterosgel, an intestinal adsorbent approved for use in IBS-D and available over-the-counter in the UK is currently the subject of a multicentre RCT in IBS-D. ${ }^{295}$ For IBS-C, there are ongoing trials of an exo-peristalsis device. ${ }^{296}$ Future research priorities are outlined below.

\section{RESEARCH: BARRIERS, PRIORITIES AND IMPLICATIONS FOR FUTURE STUDY DESIGN}

Current treatments for IBS are often inadequate and many patients remain unsatisfied with medical care. ${ }^{919295}$ Despite this, and the high prevalence of IBS, the pipeline of new treatments is relatively poor. This is related to several factors, including the challenges of running large RCTs, high thresholds for licensing of therapies and relatively low levels of academic funding, as IBS is not viewed as a priority by funders. ${ }^{297}$ The negligible mortality associated with IBS, ${ }^{149}$ together with the association with common mental disorders, ${ }^{119}$ and the stigmatisation of the condition, ${ }^{106} 107$ likely contribute to the latter. More research is required to understand the burden of suffering of patients with IBS and the direct consequences to daily life. With such a prevalent condition, it should be relatively straightforward to recruit to large RCTs, but these have often recruited slowly, and sometimes failed. This leads to a waste of both resources and participant time invested. The consequent failure to evaluate treatment efficacy adequately limits availability of potentially useful therapies.

These failings also have indirect effects, leading to reduced confidence of funders and sponsors, making trials of other therapies more challenging. There are three critical reasons for difficulty recruiting to IBS trials. First, highly restrictive inclusion criteria reduce the eligible population. Among individuals who believe themselves to have IBS, only around $60 \%$ fulfil Rome IV criteria, whereas $80 \%$ fulfil Rome III. ${ }^{23}$ There are often severity criteria built in, which aim to exclude the most severe or refractory symptoms, as well as less severe cases. In addition, many treatments are restricted to patients with IBS-C or IBS-D, whereas those with IBS-M or IBS-U are rarely recruited; indeed, although there are established composite end points for IBS-C and IBS-D, these do not exist for IBS-M or IBS-U. Second, recruitment to trials is optimal when patients are linked to specialist clinics. However, patients with IBS are widely disseminated, mostly in community care, often with no long-term follow-up, and only a minority attend specialist clinics where research is undertaken. This latter group is often more complex, more refractory and, by definition, less appropriate for treatment trials. Third, patient and public involvement exercises have identified that trial protocols are onerous and unrealistic. There are often too many visits, a need for invasive investigations, and a high burden of data collection. Patients who enter IBS trials have usually tried all available treatments and remain unsatisfied with care, so being randomised to 'standard care' for $\geq 12$ weeks is unappealing.

Future trial design needs to take these issues into consideration, using a pragmatic and participant-focused approach. Inclusion criteria should be consistent with the population that will receive the treatment. If studying a safe over-the-counter treatment, restricting inclusion to only those meeting Rome IV criteria is questionable. As with our recommendations for diagnosis, emphasising a more pragmatic clinical definition of IBS, together with limited need for investigations, the same principles should apply to trial eligibility. This would allow faster recruitment to RCTs, provide equity of access for patients and give a better indication of the true benefit of treatments being studied in the population most likely to use them. Trials should include an open-label phase, wherever possible, to allow access to the active treatment for all participants, with data collection limited to that necessary to prove efficacy and avoid use of multiple secondary academic objectives.

Recruitment methods need to include community-based approaches, involving primary care settings, social media campaigns and consent-for-contact registries. The geographical exclusion produced by site-dependent recruitment can be overcome by a remote access, or virtual, approach. ${ }^{298}$ IBS research is leading the way in this regard with the first UK interventional virtual trial being conducted in IBS, demonstrating that virtual recruitment methods outperform site-dependent recruitment significantly. ${ }^{295} 299$ Although RCTs remain the gold standard, they have many weaknesses, and modern methods of trial design may be more suitable to studying IBS. The use of virtual controls, artificial intelligence and big data solutions, together with meaningful real-time outcome data should be considered. Some important areas of research are listed below, but this is not exhaustive, will change over time and needs to be informed by a priority-setting partnership.

\section{Recommendations}

- Successful completion of large clinical trials will require pragmatic inclusion criteria, minimisation of the participant trial burden and effective recruitment strategies that reach into community settings. Virtual (remote access) trial approaches will reduce geographical exclusion.

- A priority-setting partnership would best discern valuable research questions.

- Some future research themes include, but are not limited to: - Characterisation of the illness to understand predictors (clinical, genetic, psychological and biological) of outcome and treatment response, determinants of refractory illness and burden of illness (particularly with respect to workplace productivity) by conducting large-scale epidemiological studies with extended observation.

- Trials of novel treatments, including pharmacological, dietary and behavioural therapies, device-based treatments and faecal microbiota transplantation. There is also a need for development of visceral analgesics. Consideration should be given to stratifying randomised controlled trials by IBS severity and subtype, burden of extraintestinal symptoms and psychological comorbidity.

- A better understanding of treatment combinations to uncover augmentation effects between therapies, and to assess the value of multidisciplinary approaches.

- Modulation of pain and psychological responses using pharmacological (eg, serotonin norepinephrine reuptake inhibitors) or behavioural approaches (eg, cognitive behavioural therapy used earlier in the disease course or via digital provision), and comparison of cognitive behavioural therapy with gut-directed hypnotherapy.

- Med-tech approaches (web-based, apps and devices) to behavioural modification.

\section{CONCLUSIONS}

This guideline has summarised current evidence regarding the diagnosis and management of IBS and is intended to be a practical guide for clinicians seeing patients with the condition. IBS 
is a multifactorial disorder of gut-brain interaction, and the evidence summarised here underlines the importance of effective communication, making a positive diagnosis, and instituting appropriate, evidence-based non-pharmacological and pharmacological therapies according to predominant symptoms, global patient assessment and patient choice, in order to improve both symptoms and quality of life within a bio-psychosocial framework. This guideline has also highlighted emerging new therapeutic options for IBS and priority areas for ongoing research.

\section{Author affiliations}

'Neurogastroenterology Unit, Gastroenterology, Wythenshawe Hospital, Manchester University NHS Foundation Trust, Manchester, UK

${ }^{2}$ Division of Diabetes, Endocrinology and Gastroenterology, University of Manchester, Manchester, UK

${ }^{3}$ Gastroenterology, Salford Royal Foundation Trust, Salford, UK

${ }^{4}$ Leeds Gastroenterology Institute, Leeds Teaching Hospitals NHS Trust, Leeds, UK

5 Leeds Institute of Medical Research at St James's, University of Leeds, Leeds, UK

${ }^{6}$ Gastroenterology and Hepatology, Mayo Clinic, Jacksonville, Florida, USA

${ }^{7}$ Primary Care and Population Sciences, University of Southampton, Southampton, UK

${ }^{8}$ Nottingham Digestive Diseases Biomedical Research Unit, University of Nottingham, Nottingham, UK

${ }^{9}$ Gastroenterology, Doncaster and Bassetlaw Hospitals NHS Trust, Armthorpe Road,

Doncaster, UK

${ }^{10}$ Academic Unit of Gastroenterology, Sheffield Teaching Hospitals NHS Foundation

Trust, Sheffield, UK

${ }^{11}$ Department of Infection, Immunity and Cardiovascular Disease, University of

Sheffield, Sheffield, UK

${ }^{12}$ Department of Gastroenterology, University Hospitals of North Midlands NHS Trust, Stoke-on-Trent, UK

${ }^{13}$ School of Medicine, Keele University, Keele, UK

${ }^{14}$ Department of Gastroenterology, University of Edinburgh, Western General Hospital, Edinburgh, UK

${ }^{15}$ Department of Psychology, Institute of Psychiatry, Psychology and Neuroscience, King's College London, London, UK

${ }^{16}$ Department of Gastroenterology, County Durham and Darlington NHS Foundation Trust, Durham, UK

Correction notice This article has been corrected since it published Online First. The author affiliations have been updated.

Twitter Dipesh H Vasant @DipeshVasant:, Christopher J Black @DrCJBlack and Alexander C Ford @alex_ford12399

Acknowledgements We are grateful to Julie Thompson, Information Manager at Guts UK, and Margaret Elizabeth Ogden, Michael Prior, John Shaw and Stevie Vaneghan from the Patient Advisory Group, Nottingham Biomedical Research Centre and Nottingham University Hospitals NHS Trust, Gastrointestinal and Liver Theme, for reading this guideline and providing feedback from the patient perspective.

Contributors DHV, PAP, CJB, LAH, HE, MC, AA, IA, ADF, MPE, RM-M, YY and ACF conceived and drafted the study. All authors drafted the manuscript. ACF, DHV and PAP edited the manuscript. All authors commented on drafts of the manuscript. All authors have approved the final draft of the manuscript.

Funding The authors have not declared a specific grant for this research from any funding agency in the public, commercial or not-for-profit sectors.

Competing interests Please see the attached supplement of declarations of conflicts of interest for all authors.

Patient consent for publication Not required.

Provenance and peer review Not commissioned; externally peer reviewed.

Supplemental material This content has been supplied by the author(s). It has not been vetted by BMJ Publishing Group Limited (BMJ) and may not have been peer-reviewed. Any opinions or recommendations discussed are solely those of the author(s) and are not endorsed by BMJ. BMJ disclaims all liability and responsibility arising from any reliance placed on the content. Where the content includes any translated material, BMJ does not warrant the accuracy and reliability of the translations (including but not limited to local regulations, clinical guidelines, terminology, drug names and drug dosages), and is not responsible for any error and/or omissions arising from translation and adaptation or otherwise.

ORCID iDs

Dipesh H Vasant http://orcid.org/0000-0002-2329-0616

Christopher J Black http://orcid.org/0000-0001-5449-3603

Lesley A Houghton http://orcid.org/0000-0002-5351-0229

Imran Aziz http://orcid.org/0000-0003-3833-2302
Alexander C Ford http://orcid.org/0000-0001-6371-4359

\section{REFERENCES}

1 Spiller R, Aziz Q, Creed F, et al. Guidelines on the irritable bowel syndrome: mechanisms and practical management. Gut 2007;56:1770-98.

2 Mearin F, Lacy BE, Chang L. Bowel disorders. Gastroenterology 2016:150:1393-407.

3 Brouwers MC, Kerkvliet K, Spithoff K, et al. The agree reporting checklist: a tool to improve reporting of clinical practice guidelines. BMJ 2016:352:i1152.

4 Howick J, Phillips B, Ball C. Oxford centre for evidence-based medicine levels of evidence. University of Oxford, Centre for Evidence-Based Medicine, 2009.

5 Guyatt G, Oxman AD, AkI EA, et al. Grade guidelines: 1. IntroductionGRADE evidence profiles and summary of findings tables. J Clin Epidemiol 2011:64:383-94

6 Barberio B, Houghton LA, Yiannakou Y, et al. Symptom stability in Rome IV vs Rome III irritable bowel syndrome. Am J Gastroenterol 2021:116:362-71.

7 Ford AC, Bercik P, Morgan DG, et al. Characteristics of functional bowel disorder patients: a cross-sectional survey using the Rome III criteria. Aliment Pharmacol Ther 2014;39:312-21

8 Longstreth GF, Thompson WG, Chey WD, et al. Functional bowel disorders. Gastroenterology 2006;130:1480-91

9 Palsson OS, Whitehead WE, van Tilburg MAL, et al. Development and validation of the Rome IV diagnostic questionnaire for adults. Gastroenterology 2016;150:1481-91.

10 Hookway C, Buckner S, Crosland P, et al. Irritable bowel syndrome in adults in primary care: summary of updated NICE guidance. BMJ 2015:350:h701.

11 Lovell RM, Ford AC. Global prevalence of and risk factors for irritable bowel syndrome: a meta-analysis. Clinical Gastroenterology and Hepatology 2012;10:712-21.

12 Ford AC, Forman D, Bailey AG, et al. Irritable bowel syndrome: a 10-yr natural history of symptoms and factors that influence consultation behavior. Am J Gastroenterol 2008; 103:1229-39.

13 Pace F, Molteni P, Bollani S, et al. Inflammatory bowel disease versus irritable bowel syndrome: a hospital-based, case-control study of disease impact on quality of life. Scand J Gastroenterol 2003;38:1031-8.

14 Frändemark Åsa, Törnblom H, Jakobsson S, et al. Work productivity and activity impairment in irritable bowel syndrome (IBS): a multifaceted problem. Am J Gastroentero/ 2018:113:1540-9.

15 Flacco ME, Manzoli L, De Giorgio R. Costs of irritable bowel syndrome in European countries with universal healthcare coverage: a meta-analysis. Eur Rev Med Pharmacol Sci 2019;23:2986-3000.

16 Zhang F, Xiang W, Li C-Y, et al. Economic burden of irritable bowel syndrome in China. World J Gastroenterol 2016;22:10450-60

17 Peery AF, Crockett SD, Murphy CC, et al. Burden and cost of gastrointestinal, liver, and pancreatic diseases in the United States: update 2018. Gastroenterology 2019:156:254-72.

18 Sperber AD, Dumitrascu D, Fukudo S, et al. The global prevalence of IBS in adults remains elusive due to the heterogeneity of studies: a Rome Foundation working team literature review. Gut 2017:66:1075-82.

19 Sperber AD, Bangdiwala SI, Drossman DA, et al. Worldwide prevalence and burden of functional gastrointestinal disorders, results of Rome Foundation global study. Gastroenterology 2021;160:99-114

20 Oka P, Parr H, Barberio B, et al. Global prevalence of irritable bowel syndrome according to Rome III or IV criteria: a systematic review and meta-analysis. Lancet Gastroenterol Hepatol 2020;5:908-17.

21 Black CJ, Craig 0, Gracie DJ, et al. Comparison of the Rome IV criteria with the Rome III criteria for the diagnosis of irritable bowel syndrome in secondary care. Gut 2021;70:1110-6.

22 Vork L, Weerts ZZRM, Mujagic Z, et al. Rome III vs Rome IV criteria for irritable bowel syndrome: a comparison of clinical characteristics in a large cohort study. Neurogastroenterology \& Motility 2018;30:e13189.

23 Black CJ, Yiannakou Y, Houghton LA, et al. Epidemiological, clinical, and psychological characteristics of individuals with self-reported irritable bowel syndrome based on the Rome IV vs Rome III criteria. Clin Gastroenterol Hepatol 2020;18:392-8

24 Aziz I, Törnblom H, Palsson OS, et al. How the change in IBS criteria from Rome III to Rome IV impacts on clinical characteristics and key pathophysiological factors. Am J Gastroenterol 2018:113:1017-25.

25 Chitkara DK, van Tilburg MAL, Blois-Martin N, et al. Early life risk factors that contribute to irritable bowel syndrome in adults: a systematic review. Am J Gastroenterol 2008;103:765-74.

26 Saito YA, Talley NJ. Genetics of irritable bowel syndrome. Am J Gastroenterol 2008;103:2100-4

27 Spiller R, Garsed K. Postinfectious irritable bowel syndrome. Gastroenterology 2009:136:1979-88.

28 Meaney MJ, Szyf M. Environmental programming of stress responses through DNA methylation: life at the interface between a dynamic environment and a fixed genome. Dialogues Clin Neurosci 2005;7:103-23. 
29 Van Oudenhove L, Levy RL, Crowell MD, et al. Biopsychosocial aspects of functional gastrointestinal disorders: how central and environmental processes contribute to the development and expression of functional gastrointestinal disorders. Gastroenterology 2016;150:1355-67.

30 Windgassen S, Moss-Morris R, Chilcot J, et al. The journey between brain and gut: a systematic review of psychological mechanisms of treatment effect in irritable bowel syndrome. Br J Health Psychol 2017;22:701-36.

31 Koloski NA, Jones M, Talley NJ. Evidence that independent gut-to-brain and brain-to-gut pathways operate in the irritable bowel syndrome and functional dyspepsia: a 1-year population-based prospective study. Aliment Pharmacol Ther 2016;44:592-600.

32 Drossman DA, Hasler WL. Rome IV-functional Gi disorders: disorders of gut-brain interaction. Gastroenterology 2016;150:1257-61.

33 Mayer EA, Labus J, Aziz Q, et al. Role of brain imaging in disorders of brain-gut interaction: a Rome working team report. Gut 2019;68:1701-15.

34 Black CJ, Yiannakou Y, Houghton LA. Anxiety-related factors associated with symptom severity in irritable bowel syndrome. Neurogastroenterology and motility : the official journal of the European Gastrointestinal Motility Society 2020:e13872.

35 Heymen S, Maixner W, Whitehead WE, et al. Central processing of noxious somatic stimuli in patients with irritable bowel syndrome compared with healthy controls. Clin J Pain 2010;26:104-9.

36 Berman SM, Chang L, Suyenobu B, et al. Condition-specific deactivation of brain regions by 5-HT3 receptor antagonist Alosetron. Gastroenterology 2002;123:969-77.

37 Morgan Vet al. Amitriptyline reduces rectal pain related activation of the anterior cingulate cortex in patients with irritable bowel syndrome. Gut 2005;54:601-7.

38 Lackner JM, Lou Coad M, Mertz HR, et al. Cognitive therapy for irritable bowel syndrome is associated with reduced limbic activity, Gi symptoms, and anxiety. Behav Res Ther 2006;44:621-38.

39 Salvioli B, Pellegatta G, Malacarne $M$, et al. Autonomic nervous system dysregulation in irritable bowel syndrome. Neurogastroenterology \& Motility 2015;27:423-30.

40 Bonaz B, Bazin T, Pellissier S. The vagus nerve at the interface of the microbiota-gutbrain axis. Front Neurosci 2018;12:49.

41 Sanger GJ, Chang L, Bountra C, et al. Challenges and prospects for pharmacotherapy in functional gastrointestinal disorders. Therap Adv Gastroenterol 2010;3:291-305.

42 Simrén M, Törnblom H, Palsson OS, et al. Visceral hypersensitivity is associated with Gi symptom severity in functional $\mathrm{Gi}$ disorders: consistent findings from five different patient cohorts. Gut 2018;67:255-62

43 Agrawal A, Houghton LA, Lea R, et al. Bloating and distention in irritable bowe syndrome: the role of visceral sensation. Gastroenterology 2008;134:1882-9.

44 Camilleri M, McKinzie S, Busciglio I, et al. Prospective study of motor, sensory, psychologic, and autonomic functions in patients with irritable bowel syndrome. Clin Gastroenterol Hepatol 2008;6:772-81.

45 Törnblom H, Van Oudenhove L, Sadik R, et al. Colonic Transit Time and IBS Symptoms: What's the Link? Am J Gastroentero/ 2012;107:754-60.

46 Agrawal A, Houghton LA, Reilly B, et al. Bloating and distension in irritable bowel syndrome: the role of gastrointestinal transit. Am J Gastroenterol 2009; 104:1998-2004.

47 Sadik R, Stotzer P-o, Simrén M, et al. Gastrointestinal transit abnormalities are frequently detected in patients with unexplained $\mathrm{Gi}$ symptoms at a tertiary centre. Neurogastroenterology \& Motility 2008;20:197-205.

48 Ravi K, Bharucha AE, Camilleri M, et al. Phenotypic variation of colonic motor functions in chronic constipation. Gastroenterology 2010;138:89-97.

49 houghton la, atkinson w, lockhart s, et al. Sigmoid-colonic motility in health and irritable bowel syndrome: a role for 5-hydroxytryptamine. Neurogastroenterology \& Motility 2007;19:724-31.

50 Atkinson W, Lockhart S, Whorwell PJ, et al. Altered 5-hydroxytryptamine signaling in patients with Constipation- and diarrhea-predominant irritable bowel syndrome. Gastroenterology 2006;130:34-43.

51 Klem F, Wadhwa A, Prokop LJ, et al. Prevalence, risk factors, and outcomes of irritable bowel syndrome after infectious enteritis: a systematic review and meta-analysis. Gastroenterology 2017;152:1042-54.

52 Martin-Viñas JJ, Quigley EMM. Immune response in irritable bowel syndrome: a systematic review of systemic and mucosal inflammatory mediators. J Dig Dis 2016;17:572-81.

53 Burns G, Carroll G, Mathe A, et al. Evidence for local and systemic immune activation in functional dyspepsia and the irritable bowel syndrome: a systematic review. Am J Gastroenterol 2019:114:429-36.

54 Robles A, Perez Ingles D, Myneedu K, et al. Mast cells are increased in the small intestinal mucosa of patients with irritable bowel syndrome: a systematic review and meta-analysis. Neurogastroenterology \& Motility 2019;31:e13718.

55 Wouters MM, Vicario M, Santos J. The role of mast cells in functional Gi disorders. Gut 2016;65:155-68

56 Barbara G, Stanghellini V, De Giorgio R, et al. Activated mast cells in proximity to colonic nerves correlate with abdominal pain in irritable bowel syndrome. Gastroenterology 2004;126:693-702.
57 Park JHO, Rhee PL, Kim HSEO, et al. Mucosal mast cell counts correlate with visceral hypersensitivity in patients with diarrhea predominant irritable bowel syndrome. J Gastroenterol Hepatol 2006;21:71-8.

58 Braak B, Klooker TK, Wouters MM, et al. Mucosal immune cell numbers and visceral sensitivity in patients with irritable bowel syndrome: is there any relationship? Am J Gastroenterol 2012;107:715-26.

59 Gao J. Correlation between anxiety-depression status and cytokines in diarrheapredominant irritable bowel syndrome. Exp Ther Med 2013;6:93-6.

60 Spiller RCet al. Increased rectal mucosal enteroendocrine cells, T lymphocytes, and increased gut permeability following acute Campylobacter enteritis and in postdysenteric irritable bowel syndrome. Gut 2000;47:804-11.

61 Dunlop SP, Hebden J, Campbell E, et al. Abnormal intestinal permeability in subgroups of diarrhea-predominant irritable bowel syndromes. Am J Gastroenterol 2006;101:1288-94.

62 Zhou Q, Zhang B, Verne NG. Intestinal membrane permeability and hypersensitivity in the irritable bowel syndrome. Pain 2009;146:41-6.

63 Piche T. Tight junctions and IBS - the link between epithelial permeability, lowgrade inflammation, and symptom generation? Neurogastroenterology \& Motility 2014:26:296-302.

64 Piche T, Barbara G, Aubert P, et al. Impaired intestinal barrier integrity in the colon of patients with irritable bowel syndrome: involvement of soluble mediators. Gut 2009;58:196-201.

65 Peters SA, Edogawa S, Sundt WJ, et al. Constipation-Predominant irritable bowe syndrome females have normal colonic barrier and secretory function. Am J Gastroenterol 2017:112:913-23.

66 Martínez C, Vicario M, Ramos L, et al. The jejunum of diarrhea-predominant irritable bowel syndrome shows molecular alterations in the tight junction signaling pathway that are associated with mucosal pathobiology and clinical manifestations. Am J Gastroentero/ 2012;107:736-46.

67 Fritscher-Ravens A, Schuppan D, Ellrichmann M, et al. Confocal endomicroscopy shows food-associated changes in the intestinal mucosa of patients with irritable bowel syndrome. Gastroenterology 2014;147:1012-20.

68 Fritscher-Ravens A, Pflaum T, Mösinger M, et al. Many patients with irritable bowe syndrome have atypical food allergies not associated with immunoglobulin $\mathrm{E}$. Gastroenterology 2019;157:109-18.

69 Villarreal AA, Aberger FJ, Benrud R. Use of broad-spectrum antibiotics and the development of irritable bowel syndrome. WMJ 2012;111:17-20.

70 Pimentel $M$, Lembo $A$, Chey WD, et al. Rifaximin therapy for patients with irritable bowel syndrome without constipation. N Engl J Med Overseas Ed 2011:364:22-32.

71 Bhattarai Y, Muniz Pedrogo DA, Kashyap PC. Irritable bowel syndrome: a gut microbiota-related disorder? Am J Physiol Gastrointest Liver Physiol 2017;312:G52-62.

72 grover $m$, kanazawa m, palsson os, et al. Small intestinal bacterial overgrowth in irritable bowel syndrome: association with colon motility, bowel symptoms, and psychological distress. Neurogastroenterology \& Motility 2008;20:998-1008.

73 Aguilera-Lizarraga J, Florens MV, Viola MF, et al. Local immune response to food antigens drives meal-induced abdominal pain. Nature 2021:590:151-6.

74 Hugerth LW, Andreasson A, Talley NJ, et al. No distinct microbiome signature of irritable bowel syndrome found in a Swedish random population. Gut 2020;69:1076-84.

75 Tap J, Derrien M, Törnblom H, et al. Identification of an intestinal microbiota signature associated with severity of irritable bowel syndrome. Gastroenterology 2017;152:111-23.

76 Mars RAT, Yang Y, Ward T, et al. Longitudinal multi-omics reveals subset-specific mechanisms underlying irritable bowel syndrome. Cell 2020;182:1460-73.

77 Boeckxstaens G, Camilleri M, Sifrim D, et al. Fundamentals of neurogastroenterology: Physiology/Motility - sensation. Gastroenterology 2016;150:1292-304.

78 Gazouli M, Wouters MM, Kapur-Pojskić L, et al. Lessons learned — resolving the enigma of genetic factors in IBS. Nat Rev Gastroenterol Hepatol 2016;13:77-87.

79 Czogalla B, Schmitteckert S, Houghton LA, et al. A meta-analysis of immunogenetic case-control association studies in irritable bowel syndrome. Neurogastroenterol Motil 2015:27:717-27

80 Mahurkar-Joshi S, Chang L. Epigenetic mechanisms in irritable bowel syndrome. Front Psychiatry 2020:11:805

81 Soubieres A, Wilson P, Poullis A, et al. Burden of irritable bowel syndrome in an increasingly cost-aware National health service. Frontline Gastroenterol 2015;6:246-51

82 Thompson WG, Heaton K, Smyth GT CS. Irritable bowel syndrome in general practice: prevalence, characteristics, and referral. Gut 2000;46:78-82.

83 May C, Allison G, Chapple A, et al. Framing the doctor-patient relationship in chronic illness: a comparative study of general practitioners' accounts. Sociol Health IIIn 2004; $26: 135-58$

84 National Institute for Health and Care Excellence. Suspected cancer: recognition and referral NICE guideline [NG12], 2015. Available: https://wwwniceorguk/ guidance/ng12/chapter/1-recommendations-organised-by-site-of-cancer\#lowergastrointestinal-tract-cancers 
85 Hamilton W, Peters TJ, Bankhead C, et al. Risk of ovarian cancer in women with symptoms in primary care: population based case-control study. BMJ 2009;339:b2998.

86 Everitt HA, Landau S, O'Reilly G. Assessing telephone-delivered cognitivebehavioural therapy (CBT) and web-delivered CBT versus treatment as usual in irritable bowel syndrome (ACTIB): a multicentre randomised trial. Gut 2019;68:1613-23.

87 Sibelli A, Moss-Morris R, Chalder T, et al. Patients' perspectives on GP interactions after cognitive behavioural therapy for refractory IBS: a qualitative study in UK primary and secondary care. Br J Gen Pract 2018;68:e654-62.

88 National Institute for Health and Care Excellence. Irritable bowel syndrome in adults. Quality standard [QS114], 2016. Available: https://wwwniceorguk/ guidance/qs114/chapter/Quality-statement-4-Reviewing-treatment-andmanagement

89 Ringstrom G, Sjovall H, Simrén M, et al. The importance of a Person-Centered approach in diagnostic Workups of patients with irritable bowel syndrome. Gastroenterology Nursing 2013;36:443-51.

90 Black CJ, Drossman DA, Talley NJ, et al. Functional gastrointestinal disorders: advances in understanding and management. The Lancet 2020;396:1664-74.

91 Jakobsson Ung E, Ringstrom G, Sjövall H, et al. How patients with long-term experience of living with irritable bowel syndrome manage illness in daily life. Eur J Gastroenterol Hepatol 2013;25:1478-83.

92 Bertram S, Kurland M, Lydick E. The patient's perspective of irritable bowel syndrome. J Fam Pract 2001;50:521-5.

93 Hakanson C, life E. Healthcare, and self-care management among people with irritable bowel syndrome: an integrative review of qualitative research. Gastroenterol Nurs 2014;37:217-25.

94 Casiday RE, Hungin APS, Cornford CS, et al. Patients' explanatory models for irritable bowel syndrome: symptoms and treatment more important than explaining aetiology. Fam Pract 2009;26:40-7.

95 Farndale R, Roberts L. Long-Term impact of irritable bowel syndrome: a qualitative study. Prim Health Care Res Dev 2011;12:52-67.

96 Teasdale $\mathrm{E}, \mathrm{Clarke} \mathrm{H}$, Chen N. Online forum users' views and experiences of managing irritable bowel syndrome: A qualitative analysis of discussion content. BMJ Open 2020;26:40-7.

97 Thompson GW, Heaton KW, Smyth TG, et al. Irritable bowel syndrome: the view from general practice. Eur J Gastroenterol Hepatol 1997;9:689-92.

98 Boyce PM, Koloski NA, Talley NJ. Irritable bowel syndrome according to varying diagnostic criteria: are the new Rome II criteria unnecessarily restrictive for research and practice? Am J Gastroenterol 2000;95:3176-83.

99 Mearin, X. Badía, A. Balboa, E. Bar F, Mearin F, et al. Irritable bowel syndrome prevalence varies enormously depending on the employed diagnostic criteria: comparison of Rome II versus previous criteria in a general population. Scand J Gastroentero/ 2001;36:1155-61.

100 Sanders DS, Carter MJ, Hurlstone DP, et al. Association of adult coeliac disease with irritable bowel syndrome: a case-control study in patients fulfilling Rome II criteria referred to secondary care. Lancet 2001;358:1504-8.

101 Irvine AJ, Chey WD, Ford AC. Screening for celiac disease in irritable bowel syndrome: an updated systematic review and meta-analysis. Am J Gastroenterol 2017:112:65-76

102 Muris JWM, Starmans R, Wolfs G, et al. The diagnostic value of rectal examination. Fam Pract 1993;10:34-7.

103 The new faecal calprotectin care pathway, 2018. Available: https://wwwniceorguk/ sharedlearning/the-new-faecal-calprotectin-care-pathway

104 National Institute for Health and Care Excellence. Quantitative faecal immunochemical tests to guide referral for colorectal cancer in primary care, 2017. Available: https://wwwniceorguk/guidance/DG30

105 Shivaji UN, Ford AC. Prevalence of functional gastrointestinal disorders among consecutive new patient referrals to a gastroenterology clinic. Frontline Gastroenterol 2014;5:266-71.

106 Hearn M, Whorwell PJ, Vasant DH. Stigma and irritable bowel syndrome: a taboo subject? The Lancet Gastroenterology \& Hepatology 2020;5:607-15.

107 Feingold JH, Drossman DA. Deconstructing stigma as a barrier to treating DGBI: lessons for clinicians. Neurogastroenterology \& Motility;33:e14080.

108 Drossman DA. 2012 David sun Lecture: helping your patient by helping YourselfHow to improve the Patient-Physician relationship by optimizing communication skills. Am J Gastroenterol 2013;108:521-8.

109 Langewitz Wet al. Spontaneous talking time at start of consultation in outpatient clinic: cohort study. BMJ 2002;325:682-3.

110 Hulme K, Chilcot J, Smith MA. Doctor-Patient relationship and quality of life in irritable bowel syndrome: an exploratory study of the potential mediating role of illness perceptions and acceptance. Psychol Health Med 2018;23:674-84.

111 Owens DM, Nelson DK, Talley NJ. The irritable bowel syndrome: long-term prognosis and the physician-patient interaction. Ann Intern Med 1995;122:107-12.

112 Card T, Enck P, Barbara G, et al. Post-infectious IBS: defining its clinical features and prognosis using an internet-based survey. United European Gastroenterol. j. 2018;6:1245-53.
113 Heaton KW, Ghosh S, Braddon FE. How bad are the symptoms and bowel dysfunction of patients with the irritable bowel syndrome? A prospective, controlled study with emphasis on stool form. Gut 1991;32:73-9.

114 Houghton LA, Lea R, Agrawal A, et al. Relationship of abdominal bloating to distention in irritable bowel syndrome and effect of bowel habit. Gastroenterology 2006:131:1003-10.

115 Lovell RM, Ford AC. Prevalence of gastro-esophageal reflux-type symptoms in individuals with irritable bowel syndrome in the community: a meta-analysis. Am J Gastroenterol 2012;107:1793-801

116 Ford AC, Marwaha A, Lim A, et al. Systematic review and meta-analysis of the prevalence of irritable bowel syndrome in individuals with dyspepsia. Clinical Gastroenterology and Hepatology 2010;8:401-9.

117 Aziz I, Palsson OS, Törnblom H, et al. The prevalence and impact of overlapping Rome IV-diagnosed functional gastrointestinal disorders on somatization, quality of life, and healthcare utilization: a cross-sectional general population study in three countries. Am J Gastroenterol 2018;113:86-96.

118 Petersen MW, Schröder A JT, Ørnbøl E, et al. The unifying diagnostic construct of bodily distress syndrome (BDS) was confirmed in the general population. $J$ Psychosom Res 2020;128:109868

119 Zamani M, Alizadeh-Tabari S, Zamani V. Systematic review with meta-analysis: the prevalence of anxiety and depression in patients with irritable bowel syndrome. Aliment Pharmacol Ther 2019:50:132-43.

120 Patel P, Bercik P, Morgan DG, et al. Irritable bowel syndrome is significantly associated with somatisation in 840 patients, which may drive bloating. Aliment Pharmacol Ther 2015;41:449-58.

121 Longstreth GF, Yao JF. Irritable bowel syndrome and surgery: a multivariable analysis is. Gastroenterology 2004;126:1665-73.

122 Ford AC, Veldhuyzen van Zanten SJO, Rodgers CC, et al. Diagnostic utility of alarm features for colorectal cancer: systematic review and meta-analysis. Gut 2008; 57:1545-53.

123 Whitehead WE, Palsson OS, Feld AD, et al. Utility of red flag symptom exclusions in the diagnosis of irritable bowel syndrome. Aliment Pharmacol Ther 2006:24:137-46.

124 Black CJ, Ford AC. Rational investigations in irritable bowel syndrome. Frontline Gastroenterol 2020;11:140-7.

125 Chey WD, Nojkov B, Rubenstein JH, et al. The yield of colonoscopy in patients with non-constipated irritable bowel syndrome: results from a prospective, controlled us trial. Am J Gastroenterol 2010;105:859-65.

126 Spiegel BMR, Gralnek IM, Bolus R, et al. Is a negative colonoscopy associated with reassurance or improved health-related quality of life in irritable bowel syndrome? Gastrointest Endosc 2005;62:892-9.

127 Macaigne G, Lahmek P, Locher C, et al. Microscopic colitis or functional bowel disease with diarrhea: a French prospective multicenter study. Am J Gastroenterol 2014:109:1461-70.

128 Beaugerie L, Pardi DS. Review article: drug-induced microscopic colitis - proposal for a scoring system and review of the literature. Aliment Pharmacol Ther 2005;22:277-84

129 Kane JS, Rotimi 0, Everett SM, et al. Development and validation of a scoring system to identify patients with microscopic colitis. Clinical Gastroenterology and Hepatology 2015:13:1125-31.

130 Khalid U, Lalji A, Stafferton R, et al. Bile acid malabsoption: a forgotten diagnosis? Clin Med 2010;10:124-6.

131 Arasaradnam RP, Brown S, Forbes A, et al. Guidelines for the investigation of chronic diarrhoea in adults: British Society of gastroenterology, 3rd edition. Gut 2018:67:1380-99.

132 Orekoya O, McLaughlin J, Leitao E, et al. Quantifying bile acid malabsorption helps predict response and tailor sequestrant therapy. Clin Med 2015;15:252-7.

133 Aziz I, Mumtaz S, Bholah H, et al. High prevalence of idiopathic bile acid diarrhea among patients with diarrhea-predominant irritable bowel syndrome based on Rome III criteria. Clinical Gastroenterology and Hepatology 2015;13:1650-5.

134 Slattery SA, Niaz O, Aziz O, et al. Systematic review with meta-analysis: the prevalence of bile acid malabsorption in the irritable bowel syndrome with diarrhoea. Aliment Pharmacol Ther 2015;42:3-11.

135 Wedlake L, A'Hern R, Russell D, et al. Systematic review: the prevalence of idiopathic bile acid malabsorption as diagnosed by SeHCAT scanning in patients with diarrhoea-predominant irritable bowel syndrome. Aliment Pharmacol Ther 2009;30:707-17.

136 Suttor VP, Prott GM, Hansen RD, et al. Evidence for pelvic floor dyssynergia in patients with irritable bowel syndrome. Dis Colon Rectum 2010;53:156-60.

137 Wong RK, Palsson OS, Turner MJ, et al. Inability of the Rome III criteria to distinguish functional constipation from constipation-subtype irritable bowel syndrome. Am J Gastroenterol 2010;105:2228-34.

138 Rao SSC, Valestin J, Brown CK, et al. Long-Term efficacy of biofeedback therapy for dyssynergic defecation: randomized controlled trial. Am J Gastroenterol 2010:105:890-6.

139 Corsetti M, Brown S, Chiarioni G. Chronic constipation in adults: contemporary perspectives and clinical challenges. 2: conservative, behavioural, medical and surgical treatment. Neurogastroenterology \& Motility. 
140 Leeds JS, Hopper AD, Sidhu R, et al. Some patients with irritable bowel syndrome may have exocrine pancreatic insufficiency. Clin Gastroenterol Hepatol 2010;8:433-8.

141 Talley NJ, Holtmann G, Nguyen QN, et al. Undiagnosed pancreatic exocrine insufficiency and chronic pancreatitis in functional Gi disorder patients with diarrhea or abdominal pain. J Gastroenterol Hepatol 2017;32:1813-7.

142 Yu D, Cheeseman F, Vanner S. Combined oro-caecal scintigraphy and lactulose hydrogen breath testing demonstrate that breath testing detects oro-caecal transit, not small intestinal bacterial overgrowth in patients with IBS. Gut 2011;60:334-40.

143 Posserud I, Stotzer P-0, Björnsson ES, et al. Small intestinal bacterial overgrowth in patients with irritable bowel syndrome. Gut 2007;56:802-8

144 Ghoshal UC, Srivastava D, Ghoshal U, et al. Breath tests in the diagnosis of small intestinal bacterial overgrowth in patients with irritable bowel syndrome in comparison with quantitative upper gut aspirate culture. Eur J Gastroenterol Hepatol 2014;26:753-60.

145 Tuteja AK, Talley NJ, Stoddard GJ, et al. Double-Blind placebo-controlled study of rifaximin and lactulose hydrogen breath test in Gulf War veterans with irritable bowel syndrome. Dig Dis Sci 2019;64:838-45.

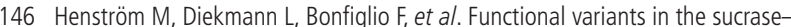
isomaltase gene associate with increased risk of irritable bowel syndrome. Gut 2018;67:263-70

147 Kim SB, Calmet FH, Garrido J, et al. Sucrase-Isomaltase deficiency as a potential masquerader in irritable bowel syndrome. Dig Dis Sci 2020;65:534-40.

148 Ringström G, Störsrud S, Posserud I, et al. Structured patient education is superior to written information in the management of patients with irritable bowel syndrome: a randomized controlled study. Eur J Gastroenterol Hepatol 2010;22:420-8.

149 Ford AC, Forman D, Bailey AG, et al. Effect of dyspepsia on survival: a longitudinal 10-year follow-up study. Am J Gastroenterol 2012;107:912-21.

150 Daley A, Grimmett C, Roberts L, et al. The effects of exercise upon symptoms and quality of life in patients diagnosed with irritable bowel syndrome: a randomised controlled trial. Int J Sports Med 2008;29:778-82.

151 Johannesson $\mathrm{E}$, Simrén $\mathrm{M}$, Strid $\mathrm{H}$, et al. Physical activity improves symptoms in irritable bowel syndrome: a randomized controlled trial. Am J Gastroenterol 2011;106:915-22.

152 Johannesson Eet al. Intervention to increase physical activity in irritable bowel syndrome shows long-term positive effects. WJG 2015;21:600-8

153 Black CJ, Burr NE, Camilleri M, et al. Efficacy of pharmacological therapies in patients with IBS with diarrhoea or mixed stool pattern: systematic review and network meta-analysis. Gut 2020;69:74-82.

154 Black CJ, Burr NE, Ford AC. Relative efficacy of tegaserod in a systematic review and network meta-analysis of licensed therapies for irritable bowel syndrome with constipation. Clinical Gastroenterology and Hepatology 2020;18:1238-9.

155 Black CJ, Burr NE, Quigley EMM, et al. Efficacy of Secretagogues in Patients With Irritable Bowel Syndrome With Constipation: Systematic Review and Network Metaanalysis. Gastroenterology 2018;155:1753-63

156 Black CJ, Thakur ER, Houghton LA, et al. Efficacy of psychological therapies for irritable bowel syndrome: systematic review and network meta-analysis. Gut 2020;69:1441-51.

157 Black CJ, Yuan Y, Selinger CP, et al. Efficacy of soluble fibre, antispasmodic drugs, and gut-brain neuromodulators in irritable bowel syndrome: a systematic review and network meta-analysis. Lancet Gastroenterol Hepatol 2020;5:117-31.

158 Ford AC, Harris LA, Lacy BE, et al. Systematic review with meta-analysis: the efficacy of prebiotics, probiotics, synbiotics and antibiotics in irritable bowel syndrome. Aliment Pharmacol Ther 2018;48:1044-60.

159 Ford AC, Lacy BE, Harris LA, et al. Effect of antidepressants and psychological therapies in irritable bowel syndrome: an updated systematic review and metaanalysis. Am J Gastroenterol 2019;114:21-39.

160 Ford AC, Moayyedi P, Chey WD, et al. American College of gastroenterology monograph on management of irritable bowel syndrome. Am J Gastroenterol 2018;113:1-18.

161 Dionne J, Ford AC, Yuan Y, et al. A systematic review and meta-analysis evaluating the efficacy of a gluten-free diet and a low FODMAPs diet in treating symptoms of irritable bowel syndrome. American Journal of Gastroenterology 2018;113:1290-300.

162 Black CJ, Moayyedi P, Quigley EMM, et al. Peppermint oil in irritable bowel syndrome. Gastroenterology 2020;159:395-6.

163 Ford AC, Brandt LJ, Young C, et al. Efficacy of 5-HT3 antagonists and 5-HT4 agonists in irritable bowel syndrome: systematic review and meta-analysis. Am J Gastroenterol 2009;104:1831-43.

164 Chang L, Lacy BE, Moshiree B, et al. Mo1550 a novel score to evaluate abdominal symptom improvement in patients with CONSTIPATION-PREDOMINANT IBS demonstrates efficacy of linaclotide for improving abdominal bloating, discomfort, and pain in a phase 3B trial. Gastroenterology 2020;158:S891.

165 Chey WD, Sayuk GS, Bartolini W, et al. Randomized trial of 2 delayed-release formulations of linaclotide in patients with irritable bowel syndrome with constipation. Am J Gastroenterol 2021;116:354-61.

166 Barraza-Ortiz DA, Pérez-López N, Medina-López VM, et al. Combination of a probiotic and an antispasmodic increases quality of life and reduces symptoms in patients with irritable bowel syndrome: a pilot study. Dig Dis 2020. doi:10.1159/000510950. [Epub ahead of print: 18 Aug 2020].

167 Andresen V, Gschossmann J, Layer P. Heat-Inactivated Bifidobacterium bifidum MIMBb75 (SYN-HI-001) in the treatment of irritable bowel syndrome: a multicentre, randomised, double-blind, placebo-controlled clinical trial. The Lancet Gastroenterology \& Hepatology 2020;5:658-66.

168 Martoni CJ, Srivastava S, Leyer GJ. Lactobacillus acidophilus DDS-1 and Bifidobacterium lactis UABla-12 Improve Abdominal Pain Severity and Symptomology in Irritable Bowel Syndrome: Randomized Controlled Trial. Nutrients 2020;12:363.

169 JH O, Jang YS, Kang D. Efficacy and safety of new Lactobacilli probiotics for unconstipated irritable bowel syndrome: A randomized, double-blind, placebocontrolled trial. Nutrients 2019;11:2887.

170 Al Helo A, Helo AA, Department of family medicine, Jordanian ministry of health, Jordan. Treatment of irritable bowel syndrome with Saccharomyces cerevisiae. J Gastroenterol Hepatol Res 2019;8:2919-22.

171 Ishaque SM, Khosruzzaman SM, Ahmed DS, et al. A randomized placebo-controlled clinical trial of a multi-strain probiotic formulation (Bio-Kult $\circledast$ ) in the management of diarrhea-predominant irritable bowel syndrome. BMC Gastroenterol 2018;18:71.

172 Shin SP, Choi YM, Kim WH, et al. A double blind, placebo-controlled, randomized clinical trial that breast milk derived-<i>Lactobacillus gasseri</i> BNR17 mitigated diarrhea-dominant irritable bowel syndrome. J Clin Biochem Nutr 2018;62:179-86.

173 Sun Y-Y, Li M, Li Y-Y, et al. The effect of Clostridium butyricum on symptoms and fecal microbiota in diarrhea-dominant irritable bowel syndrome: a randomized, doubleblind, placebo-controlled trial. Sci Rep 2018;8:2964.

174 Zhang Y, Fox M, Dai N. Low-FODMAP diet versus traditional dietary advice for diarrhea-predominant irritable bowel syndrome: a randomized, parallel-controlled trial with analysis of clinical and microbiological factors associated with patient outcome. Neurogastroenterology and motility : the official journal of the European Gastrointestinal Motility Society 2020;32

175 Wilson B, Rossi M, Kanno T, et al. $\beta$-galactooligosaccharide in conjunction with low FODMAP diet improves irritable bowel syndrome symptoms but reduces fecal Bifidobacteria. Am J Gastroenterol 2020;115:906-15.

176 Patcharatrakul T, Juntrapirat A, Lakananurak N, et al. Effect of structural individual low-FODMAP dietary advice vs. brief advice on a commonly recommended diet on IBS symptoms and intestinal gas production. Nutrients 2019;11:2856.

177 Harvie RM, Chisholm AW, Bisanz JE, et al. Long-Term irritable bowel syndrome symptom control with reintroduction of selected FODMAPs. WJG 2017;23:4632-43.

178 Plasse TF, Barton G, Davidson E, et al. Bimodal release ondansetron improves stool consistency and symptomatology in diarrhea-predominant irritable bowel syndrome: a randomized, double-blind, trial. Am J Gastroenterol 2020:115:1466-73.

179 Fukudo S, Nakamura M, Hamatani T, et al. Efficacy and safety of 5-HT4 receptor agonist minesapride for irritable bowel syndrome with constipation in a randomized controlled trial. Clinical Gastroenterology and Hepatology 2021;19:538-46.

180 Hamatani T, Fukudo S, Nakada Y, et al. Randomised clinical trial: minesapride vs placebo for irritable bowel syndrome with predominant constipation. Aliment Pharmacol Ther 2020:52:430-41.

181 Deeks JJ. Issues in the selection of a summary statistic for meta-analysis of clinical trials with binary outcomes. Stat Med 2002;21:1575-600.

182 Salanti G, Higgins JPT, Ades AE, et al. Evaluation of networks of randomized trials. Stat Methods Med Res 2008:17:279-301.

183 Salanti G. Indirect and mixed-treatment comparison, network, or multiple-treatments meta-analysis: many names, many benefits, many concerns for the next generation evidence synthesis tool. Res. Syn. Meth. 2012:3:80-97.

184 Salanti G, Ades AE, loannidis JPA. Graphical methods and numerical summaries for presenting results from multiple-treatment meta-analysis: an overview and tutorial. $J$ Clin Epidemio/ 2011;64:163-71.

185 Rücker G, Schwarzer G. Ranking treatments in frequentist network meta-analysis works without resampling methods. BMC Med Res Methodol 2015;15:58.

186 DerSimonian R, Laird N. Meta-Analysis in clinical trials. Control Clin Trials 1986:7:177-88.

187 Higgins JPT, Thompson SG. Quantifying heterogeneity in a meta-analysis. Stat Med 2002:21:1539-58.

188 Simrén M, Månsson A, Langkilde AM, et al. Food-Related gastrointestinal symptoms in the irritable bowel syndrome. Digestion 2001:63:108-15.

189 Böhn L, Störsrud S, Törnblom H, et al. Self-Reported food-related gastrointestinal symptoms in IBS are common and associated with more severe symptoms and reduced quality of life. Am J Gastroenterol 2013;108:634-41.

190 Lind R, Arslan G, Eriksen HR, et al. Subjective health complaints and modern health worries in patients with subjective food hypersensitivity. Dig Dis Sci 2005:50:1245-51.

191 Berstad A, Undseth R, Lind R, et al. Functional bowel symptoms, fibromyalgia and fatigue: a food-induced triad? Scand J Gastroenterol 2012;47:914-9.

192 Halpert A, Dalton CB, Palsson O, et al. What patients know about irritable bowe syndrome (IBS) and what they would like to know. National survey on patient educational needs in IBS and development and validation of the patient educational needs questionnaire (PEQ). Am J Gastroenterol 2007;102:1972-82. 
193 Spencer M, Chey WD, Eswaran S. Dietary renaissance in IBS: has food replaced medications as a primary treatment strategy? Curr Treat Options Gastroenterol 2014; 12:424-40.

194 Moayyedi P, Simrén M, Bercik P. Evidence-Based and mechanistic insights into exclusion diets for IBS. Nat Rev Gastroenterol Hepatol 2020;17:406-13.

195 Atkinson Wet al. Food elimination based on IgG antibodies in irritable bowe syndrome: a randomised controlled trial. Gut 2004;53:1459-64.

196 Hunter JO. Food elimination in IBS: the case for IgG testing remains doubtful. Gut2005;54:1203. author reply.

197 Ali A, Weiss TR, McKee D, et al. Efficacy of individualised diets in patients with irritable bowel syndrome: a randomised controlled trial. BMJ Open Gastroenterol 2017;4:e000164.

198 McKenzie YA, Bowyer RK, Leach H, et al. British dietetic association systematic review and evidence-based practice guidelines for the dietary management of irritable bowel syndrome in adults (2016 update). J Hum Nutr Diet 2016;29:549-75.

199 British Dietetic Association. Food fact sheet: irritable bowel syndrome and diet. Available: https://wwwbdaukcom/resourceDetail/printPdf/?resource=irritable-bowelsyndrome-diet

200 Major G, Murray K, Singh G, et al. Demonstration of differences in colonic volumes, transit, chyme consistency, and response to psyllium between healthy and constipated subjects using magnetic resonance imaging. Neurogastroenterology \& Motility 2018;30:e13400.

201 Major G, Pritchard S, Murray K, et al. Colon hypersensitivity to distension, rather than excessive gas production, produces carbohydrate-related symptoms in individuals with irritable bowel syndrome. Gastroenterology 2017;152:124-33.

202 Spiller R. Impact of diet on symptoms of the irritable bowel syndrome. Nutrients 2021; 13:575

203 O'Keeffe M, Jansen C, Martin L, et al. Long-Term impact of the low-FODMAP diet on gastrointestinal symptoms, dietary intake, patient acceptability, and healthcare utilization in irritable bowel syndrome. Neurogastroenterol Motil 2018;30. doi:10.1111/nmo.13154. [Epub ahead of print: 1407 2017]

204 Aziz I. The global phenomenon of self-reported wheat sensitivity. Am J Gastroenterol 2018;113:945-8

205 Biesiekierski JR, Peters SL, Newnham ED, et al. No effects of gluten in patients with self-reported non-celiac gluten sensitivity after dietary reduction of fermentable, poorly absorbed, short-chain carbohydrates. Gastroenterology 2013;145:320-8.

206 Skodje GI, Sarna VK, Minelle IH, et al. Fructan, rather than gluten, induces symptoms in patients with self-reported non-celiac gluten sensitivity. Gastroenterology 2018:154:529-39.

207 Rej A, Aziz I, Tornblom H, et al. The role of diet in irritable bowel syndrome: implications for dietary advice. J Intern Med 2019;286:490-502.

208 Wilson B, Cox SR, Whelan K. Challenges of the low FODMAP diet for managing irritable bowel syndrome and approaches to their minimisation and mitigation. Proc Nutr Soc 2020;29:1-10.

209 Tuck CJ, Vanner SJ. Dietary therapies for functional bowel symptoms: recent advances, challenges, and future directions. Neurogastroenterology \& Motility 2018;30:e13238.

210 Mari A, Hosadurg D, Martin L, et al. Adherence with a low-FODMAP diet in irritable bowel syndrome: are eating disorders the missing link? Eur J Gastroenterol Hepatol 2019:31:178-82

211 Melchior C, Desprez C, Riachi G, et al. Anxiety and depression profile is associated with eating disorders in patients with irritable bowel syndrome. Front. Psychiatry 2019;10:928

212 Halmos EP, Christophersen CT, Bird AR, et al. Diets that differ in their FODMAP content alter the colonic luminal microenvironment. Gut 2015;64:93-100.

213 Bennet SMP, Böhn L, Störsrud S, et al. Multivariate modelling of faecal bacterial profiles of patients with IBS predicts responsiveness to a diet low in FODMAPs. Gut 2018;67:872-81.

214 Rossi M, Aggio R, Staudacher HM, et al. Volatile organic compounds in feces associate with response to dietary intervention in patients with irritable bowel syndrome. Clinical Gastroenterology and Hepatology 2018;16:385-91.

215 Pinto-Sanchez MI, Nardelli A, Borojevic R, et al. Gluten-Free Diet Reduces Symptoms, Particularly Diarrhea, in Patients With Irritable Bowel Syndrome and Antigliadin IgG. Clinical Gastroenterology and Hepatology 2020;150.

216 Kassinen A, Krogius-Kurikka L, Mäkivuokko H, et al. The fecal microbiota of irritable bowel syndrome patients differs significantly from that of healthy subjects. Gastroenterology 2007;133:24-33.

217 Camilleri M, Ford A. Pharmacotherapy for irritable bowel syndrome. JCM 2017:6:101

218 Amato $A$, Liotta $R$, Mulè F. Effects of menthol on circular smooth muscle of human colon: analysis of the mechanism of action. Eur J Pharmacol 2014;740:295-301.

219 Weerts ZZRM, Masclee AAM, Witteman BJM, et al. Efficacy and safety of peppermint oil in a randomized, double-blind trial of patients with irritable bowel syndrome. Gastroenterology 2020;158:123-36.

220 Ford AC, Suares NC. Effect of laxatives and pharmacological therapies in chronic idiopathic constipation: systematic review and meta-analysis. Gut 2011;60:209-18

221 Awad RA, Camacho S, A randomized CS. A randomized, double-blind, placebocontrolled trial of polyethylene glycol effects on fasting and postprandial rectal sensitivity and symptoms in hypersensitive constipation-predominant irritable bowel syndrome. Colorectal Dis 2010;12:1131-8

222 Chapman RW, Stanghellini V, Geraint M, et al. Randomized clinical trial: Macrogol/PEG 3350 plus electrolytes for treatment of patients with constipation associated with irritable bowel syndrome. Am J Gastroenterol 2013:108:1508-15.

223 Gorard DA, Libby GW, Farthing MJG. Influence of antidepressants on whole gut and orocaecal transit times in health and irritable bowel syndrome. Aliment Pharmacol Ther 1994;8:159-66.

224 Lewis-Fernández R, Lam P, Lucak S, et al. An open-label pilot study of duloxetine in patients with irritable bowel syndrome and comorbid major depressive disorder. J Clin Psychopharmacol 2016;36:710-5.

225 Kaplan A, Franzen MD, Nickell PV, et al. An open-label trial of duloxetine in patients with irritable bowel syndrome and comorbid generalized anxiety disorder. Int $J$ Psychiatry Clin Pract 2014;18:11-15.

226 Drossman DA, Tack J, Ford AC, et al. Neuromodulators for functional gastrointestinal disorders (disorders of Gut-Brain interaction): a Rome Foundation working team report. Gastroenterology 2018;154:1140-71.

227 Houghton LA, Fell C, Whorwell PJ, et al. Effect of a second-generation 2 ligand (pregabalin) on visceral sensation in hypersensitive patients with irritable bowe syndrome. Gut 2007;56:1218-25.

228 Saito YA, Almazar AE, Tilkes KE, et al. Randomised clinical trial: pregabalin vs placebo for irritable bowel syndrome. Aliment Pharmacol Ther 2019;49:389-97.

229 Barshop K, Staller K. Eluxadoline in irritable bowel syndrome with diarrhea: rationale, evidence and place in therapy. Ther Adv Chronic Dis 2017;8:153-60.

230 Cash BD, Lacy BE, Schoenfeld PS, et al. Safety of eluxadoline in patients with irritable bowel syndrome with diarrhea. Am J Gastroenterol 2017;112:365-74.

231 Houghton LA, Foster JM, Whorwell PJ, Alosetron WPJ. Alosetron, a 5-HT3 receptor antagonist, delays colonic transit in patients with irritable bowel syndrome and healthy volunteers. Aliment Pharmacol Ther 2000;14:775-82.

232 Min YW, Rhee P-L. The clinical potential of ramosetron in the treatment of irritable bowel syndrome with diarrhea (IBS-D). Therap Adv Gastroenterol 2015;8:136-42.

233 Delvaux M, Louvel D, Mamet J-P, et al. Effect of alosetron on responses to colonic distension in patients with irritable bowel syndrome. Aliment Pharmacol Ther 1998;12:849-55.

234 Chang L, Chey WD, Harris L, et al. Incidence of ischemic colitis and serious complications of constipation among patients using Alosetron: systematic review of clinical trials and post-marketing surveillance data. Am J Gastroenterol 2006:101:1069-79.

235 Cole JA, Cook SF, Sands BE, et al. Occurrence of colon ischemia in relation to irritable bowel syndrome. Am J Gastroenterol 2004;99:486-91.

236 Garsed K, Chernova J, Hastings M, et al. A randomised trial of ondansetron for the treatment of irritable bowel syndrome with diarrhoea. Gut 2014;63:1617-25.

237 Gunn D, Fried R, Lalani R, et al. Treatment of irritable bowel syndrome with diarrhoea using titrated ondansetron (Triton): study protocol for a randomised controlled trial. Trials 2019:20:517.

238 Lembo A, Pimentel M, Rao SS, et al. Repeat treatment with rifaximin is safe and effective in patients with diarrhea-predominant irritable bowel syndrome. Gastroenterology 2016;151:1113-21.

239 Sarosiek I, Bashashati M, Alvarez A, et al. Lubiprostone accelerates intestinal transit and alleviates small intestinal bacterial overgrowth in patients with chronic constipation. Am J Med Sci 2016;352:231-8

240 Busby RW, Bryant AP, Bartolini WP, et al. Linaclotide, through activation of guanylate cyclase $C$, acts locally in the gastrointestinal tract to elicit enhanced intestinal secretion and transit. Eur J Pharmacol 2010:649:328-35.

241 Patwa V, Joshi A, Thadi A, et al. 967 Plecanatide, like uroguanylin, activates guanylate Cyclase-C signaling in a pH-dependent manner in T84 cells, and in murine intestinal epithelial cells and tissues. Gastroenterology 2016;150:S193-4.

242 Prather CM, Camilleri M, Zinsmeister AR, et al. Tegaserod accelerates orocecal transit in patients with constipation-predominant irritable bowel syndrome. Gastroenterology 2000;118:463-8.

243 Lackner JM, Jaccard J, Radziwon CD, et al. Durability and decay of treatment benefit of cognitive behavioral therapy for irritable bowel syndrome: 12-month follow-up. Am J Gastroenterol 2019:114:330-8.

244 Everitt H, Landau S, Little P, et al. Therapist telephone-delivered CBT and web-based CBT compared with treatment as usual in refractory irritable bowel syndrome: the ACTIB three-arm RCT. Health Technol Assess 2019;23:1-154

245 Vasant DH, Whorwell PJ. Gut-focused hypnotherapy for functional gastrointestinal disorders: evidence-base, practical aspects, and the Manchester protocol. Neurogastroenterol Motil 2019:31:e13573.

246 Palsson OS. Standardized Hypnosis Treatment for Irritable Bowel Syndrome: The North Carolina Protocol. International Journal of Clinical and Experimental Hypnosis 2006;54:51-64.

247 Simrén M, Ringström G, Björnsson ES, et al. Treatment with hypnotherapy reduces the sensory and motor component of the gastrocolonic response in irritable bowel syndrome. Psychosom Med 2004;66:233-8

248 Whorwell PJ, Houghton LA, Taylor EE, et al. Physiological effects of emotion: assessment via hypnosis. The Lancet 1992;340:69-72. 
249 Lea R, Houghton LA, Calvert EL, et al. Gut-focused hypnotherapy normalizes disordered rectal sensitivity in patients with irritable bowel syndrome. Aliment Pharmacol Ther 2003;17:635-42.

250 Lowén MBO, Mayer EA, Sjöberg M, et al. Effect of hypnotherapy and educational intervention on brain response to visceral stimulus in the irritable bowel syndrome. Aliment Pharmacol Ther 2013;37:1184-97.

251 Simon RA, Engström M, Icenhour A, et al. On functional connectivity and symptom relief after gut-directed hypnotherapy in irritable bowel syndrome: a preliminary study. J Neurogastroenterol Motil 2019;25:478-9.

252 Miller V, Carruthers HR, Morris J, et al. Hypnotherapy for irritable bowel syndrome: an audit of one thousand adult patients. Aliment Pharmacol Ther 2015;41:844-55.

253 Vasant DH, Hasan SS, Cruickshanks P, et al. Gut-focused hypnotherapy for children and adolescents with irritable bowel syndrome. Frontline Gastroenterol 2020;199:flgastro-2020-101679

254 Lindfors $\mathrm{P}$, Unge $\mathrm{P}$, Nyhlin $\mathrm{H}$, et al. Long-Term effects of hypnotherapy in patients with refractory irritable bowel syndrome. Scand J Gastroenterol 2012;47:414-21.

255 Houghton LA, Heyman DJ, Whorwell PJ. Symptomatology, quality of life and economic features of irritable bowel syndrome--the effect of hypnotherapy. Aliment Pharmacol Ther 1996;10:91-5.

256 Gonsalkorale WMet al. Long term benefits of hypnotherapy for irritable bowel syndrome. Gut 2003:52:1623-9.

257 Lindfors P, Ljótsson B, Bjornsson E, et al. Patient satisfaction after gut-directed hypnotherapy in irritable bowel syndrome. Neurogastroenterology \& Motility 2013:25:169-86.

258 Flik CE, Laan W, Zuithoff NPA, et al. Efficacy of individual and group hypnotherapy in irritable bowel syndrome (IMAGINE): a multicentre randomised controlled trial. Lancet Gastroenterol Hepatol 2019;4:20-31.

259 Hasan SS, Pearson JS, morris J, et al. SKYPE hypnotherapy for irritable bowel syndrome: effectiveness and comparison with face-to-face treatment. International Journal of Clinical and Experimental Hypnosis 2019;67:69-80.

260 Drossman DA, Chang L, Bellamy N, et al. Severity in irritable bowel syndrome: a Rome Foundation working team report. Am J Gastroenterol 2011;106:1749-59.

261 Harris LA, Heitkemper MM. Practical considerations for recognizing and managing severe irritable bowel syndrome. Gastroenterology Nurs 2012;35:12-21.

262 Olden KW, Brown AR. Treatment of the severe refractory irritable bowel patient. Curr Treat Options Gastroenterol 2006;9:324-30.

263 Francis CY, Morris J, Whorwell PJ. The irritable bowel severity scoring system: a simple method of monitoring irritable bowel syndrome and its progress. Aliment Pharmacol Ther 1997;11:395-402.

264 Ljótsson B, Jones M, Talley NJ, et al. Discriminant and convergent validity of the GSRS-IBS symptom severity measure for irritable bowel syndrome: a population study. United European Gastroenterol. j. 2020;8:284-92.

265 Drossman DA. Do psychosocial factors define symptom severity and patient status in irritable bowel syndrome? Am J Med 1999;107:41-50.

266 Manzoli L, Flacco M, Marzuillo C. Prevalence of severe irritable bowel syndrome among Italian adults. A meta-analysis. Eur Rev Med Pharmacol Sci 2017;21:5751-64.

267 Carter D, Beer-Gabel M, Derazne E, et al. The severity of symptoms related to irritable bowel syndrome is a risk factor for the misclassification of significant organic disease. J Clin Gastroenterol 2017:51:421-5.

268 Adeniji OA, Barnett CB, Di Palma JA. Durability of the diagnosis of irritable bowel syndrome based on clinical criteria. Dig Dis Sci 2004;49:572-4.

269 Keefer L, Drossman DA, Guthrie E, et al. Centrally mediated disorders of gastrointestinal pain. Gastroenterology 2016;150:1408-19.

270 Paine P, McLaughlin J, Lal S. Review article: the assessment and management of chronic severe gastrointestinal dysmotility in adults. Aliment Pharmacol Ther 2013;38:1209-29.

271 Kanuri N, Cassell B, Bruce SE, et al. The impact of abuse and mood on bowel symptoms and health-related quality of life in irritable bowel syndrome (IBS). Neurogastroenterology \& Motility 2016;28:1508-17.

272 Thakur ER, Gurtman MB, Keefer L. Representing the IBS outcome study Research Group. gender differences in irritable bowel syndrome: the interpersonal connection Neurogastroenterology and motility : the official journal of the European Gastrointestinal Motility Society 2015;27:1478-86.

273 Colucci R, Gambaccini D, Ghisu N, et al. Influence of the serotonin transporter 5HTTLPR polymorphism on symptom severity in irritable bowel syndrome. PLoS One 2013;8:e54831.

274 Simrén M, Törnblom H, Palsson OS, et al. Cumulative effects of psychologic distress, visceral hypersensitivity, and abnormal transit on patient-reported outcomes in irritable bowel syndrome. Gastroenterology 2019:157:391-402.

275 Stasi C, Caserta A, Nisita C, et al. The complex interplay between gastrointestinal and psychiatric symptoms in irritable bowel syndrome: a longitudinal assessment. J Gastroenterol Hepatol 2019;34:713-9.
276 Berens S, Engel F, Gauss A, et al. Patients with multiple functional gastrointestinal disorders (FGIDs) show increased illness severity: a cross-sectional study in a tertiary care FGID specialty clinic. Gastroenterol Res Pract 2020;2020:1-10.

277 Basnayake C, Kamm MA, Stanley A, et al. Standard gastroenterologist versus multidisciplinary treatment for functional gastrointestinal disorders (MANTRA): an open-label, single-centre, randomised controlled trial. Lancet Gastroenterol Hepatol 2020;5:890-9.

278 Masuy I, Pannemans J, Tack J. Irritable bowel syndrome: diagnosis and management. Minerva Gastroenterol Dietol 2019;66:136-50

279 Lacy BE, Everhart KK, Weiser KT, et al. IBS Patients' Willingness to Take Risks With Medications. Am J Gastroenterol 2012;107:804-9.

280 Berens S, Stroe-Kunold E, Kraus F, et al. Pilot-RCT of an integrative group therapy for patients with refractory irritable bowel syndrome (ISRCTN02977330). J Psychosom Res 2018;105:72-9.

281 Creed F, Guthrie E, Ratcliffe J, et al. Does psychological treatment help only those patients with severe irritable bowel syndrome who also have a concurrent psychiatric disorder? Aust N Z J Psychiatry 2005;39:807-15.

282 Hyphantis T, Guthrie E, Tomenson B, et al. Psychodynamic interpersonal therapy and improvement in interpersonal difficulties in people with severe irritable bowel syndrome. Pain 2009;145:196-203.

283 Nakajima A, Taniguchi S, Kurosu S, et al. Efficacy, long-term safety, and impact on quality of life of elobixibat in more severe constipation: post hoc analyses of two phase 3 trials in Japan. Neurogastroenterology \& Motility 2019;31:e13571.

284 Yiannakou Y, Agrawal A, Allen PB, et al. UK clinical experience up to 52 weeks with linaclotide for irritable bowel syndrome with constipation. Therap Adv Gastroenterol 2018;11:1756284818798791.

285 Brenner DM, Sayuk GS. Current US food and drug Administration-approved pharmacologic therapies for the treatment of irritable bowel syndrome with diarrhea. Adv Ther 2020:1-14.

286 Pearson JS, Pollard C, Whorwell PJ. Avoiding analgesic escalation and excessive healthcare utilization in severe irritable bowel syndrome: a role for intramuscula anticholinergics? Therap Adv Gastroenterol 2014;7:232-7.

287 Kilgallon E, Vasant DH, Green D, et al. Chronic continuous abdominal pain: evaluation of diagnostic features, iatrogenesis and drug treatments in a cohort of 103 patients. Aliment Pharmacol Ther 2019:49:1282-92.

288 Pampallona S, Bollini P, Tibaldi G, et al. Combined pharmacotherapy and psychological treatment for depression. Arch Gen Psychiatry 2004;61:714-9.

289 Holroyd KA, O'Donnell FJ, Stensland M, et al. Management of chronic tension-type headache with tricyclic antidepressant medication, stress management therapy, and their combination: a randomized controlled trial. JAMA 2001;285:2208-15.

290 Wouters MM, Balemans D, Van Wanrooy S, et al. Histamine receptor H1-Mediated sensitization of TRPV1 mediates visceral hypersensitivity and symptoms in patients with irritable bowel syndrome. Gastroenterology 2016;150:875-87.

291 Fukudo S, Endo Y, Hongo M, et al. Safety and efficacy of the sodium-glucose cotransporter 1 inhibitor mizagliflozin for functional constipation: a randomised, placebo-controlled, double-blind phase 2 trial. The Lancet Gastroenterology \& Hepatology 2018;3:603-13.

292 Castro J, Harrington AM, Hughes PA, et al. Linaclotide inhibits colonic nociceptors and relieves abdominal pain via guanylate Cyclase-C and extracellular cyclic guanosine 3',5'-Monophosphate. Gastroenterology 2013;145:1334-46.

293 Yacyshyn B, Ginsberg DC, Gilder K, et al. Su1930 - Safety and Efficacy of Olorinab, a Peripherally Restricted, Highly Selective, Cannabinoid Receptor 2 Agonist in a Phase 2A Study in Chronic Abdominal Pain Associated with Crohn's Disease. Gastroenterology 2019;156:S-665

294 Ianiro G, Eusebi LH, Black CJ, et al. Systematic review with meta-analysis: efficacy of faecal microbiota transplantation for the treatment of irritable bowel syndrome. Aliment Pharmacol Ther 2019;50:240-8.

295 Kemppinen A, Howell C, Allgar V, et al. Randomised, double-blind, placebo controlled multi-centre study to assess the efficacy, tolerability and safety of Enterosgel $($ in the treatment of irritable bowel syndrome with diarrhoea (IBS-D) in adults. Trials 2020:21:122

296 McClurg D, Booth L, Herrero-Fresneda I. Safety and efficacy of intermittent colonic exoperistalsis device to treat chronic constipation: a prospective multicentric clinical trial. Clin Trans/ Gastroenterol 2020;11:e00267.

297 The Lancet gastroenterology hepatology. unmet needs of patients with irritable bowel syndrome. The lancet Gastroenterology \& hepatology 2018;587.

298 Yiannakou Y. Virtual trials: transforming the landscape of clinical research. National health executive magazine January, 2021. Available: https://mag.nationalhealthex ecutive.com/publication/?m=62920\& $=691004 \& p=44 \&$ article_id $=3863994$

299 Virtual trial recruits 67\% faster led by NIHR Patient Recruitment Centre in Newcastle in collaboration with Enteromed, 2020. Available: https://www.nihr.ac.uk/news/ virtual-trial-recruits-67-faster-led-by-nihr-patient-recruitment-centre-in-newcastle-incollaboration-with-enteromed/26290 